\title{
The Geodesic Approximation for the Yang-Mills-Higgs Equations
}

\section{Stuart ${ }^{\star}$}

Mathematics Department, U.C. Davis, Davis, CA95616, USA. email: dmstuart@aztec.ucdavis.edu

Received: 10 September 1993/in revised form: 31 January 1994

\begin{abstract}
In this paper we consider the dynamics of the monopole solutions of Yang-Mills-Higgs theory on Minkowski space. The monopoles are solutions of the Yang-Mills-Higgs equations on three dimensional Euclidean space. It is of interest to understand how they evolve in time when considered as solutions of the YangMills-Higgs equations on Minkowski space-i.e. the time dependent equations. It was suggested by Manton that in certain situations the monopole dynamics could be understood in terms of geodesics with respect to a certain metric on the space of guage equivalence classes of monopoles-the moduli space. The metric is defined by taking the $L^{2}$ inner product of tangent vectors to this space. In this paper we will prove that Manton's approximation is indeed valid in the right circumstances, which correspond to the slow motion of monopoles. The metric on the moduli space of monopoles was analysed in a book by Atiyah and Hitchin, so together with the results of this paper a detailed and rigorous understanding of the low energy dynamics of monopoles in Yang-Mills-Higgs theory is obtained. The strategy of the proof is to develop asymptotic expansions using appropriate gauge conditions, and then to use energy estimates to prove their validity. For the case of monopoles to be considered here there is a technical obstacle to be overcome-when the equations are linearised about the monopole the continuous spectrum extends all the way to the origin. This is overcome by using a norm introduced by Taubes in a discussion of index theory for the Yang-Mills-Higgs functional.
\end{abstract}

\section{Introduction}

In this paper we will construct certain solutions of the Yang-Mills-Higgs equations on Minkowski space. To write these down let $\left(x_{0}, x_{1}, x_{2}, x_{3}\right)=\left(t, x_{1}, x_{2}, x_{3}\right)$ be coordinates on Minkowski space, then the dependent variables are an $s u(2)$ valued one form called the connection:

$$
A=A_{0} d t+A_{1} d x_{1}+A_{2} d x_{2}+A_{3} d x_{3}
$$

$\star$ Supported by grant DMS-9214067 from the National Science Foundation. 
and an $\operatorname{su}(2)$ valued function called the Higgs field $\Phi(t, x)$. The curvature is a two form

$$
F=\frac{1}{2} \sum_{\mu, v=0}^{3} F_{\mu \nu} d x_{\mu} \wedge d x_{\nu}
$$

where

$$
F_{\mu \nu}=\frac{\partial A_{v}}{\partial x_{v}}-\frac{\partial A_{\mu}}{\partial x_{v}}+\left[A_{\mu}, A_{v}\right]
$$

where $[\cdot, \cdot]$ means matrix commutation (see Sect. 2 and Appendix A for notation.) The Yang-Mills-Higgs equations are:

$$
\begin{gathered}
\sum_{i=1}^{3} D_{i} F_{i 0}=-\left[\Phi, D_{0} \Phi\right], \\
-D_{0} F_{j 0}+\sum_{i=1}^{3} D_{i} F_{j i}=-\left[\Phi, D_{j} \Phi\right], \\
D_{0}^{2} \Phi-\sum_{i=1}^{3} D_{i}^{2} \Phi=0,
\end{gathered}
$$

where $D_{\mu}=\left(\nabla_{A}\right)_{\mu}=\partial_{\mu}+\left[A_{\mu}, \cdot\right]$ is a covariant derivative. The equations are written out in terms of $(A, \Phi)$ in Sect. 5. An important property of the equations is gauge invariance - let $g(t, x)$ be a differentiable $\mathrm{SU}(2)$ valued function, then if $(A, \Phi)$ is a solution of Eqs. 1.1-1.3 then so is $\left(g d g^{-1}+g A g^{-1}, g \Phi g^{-1}\right)$.

A static solution is one in which $(A, \Phi)$ are independent of $t$ and $A_{0}=0$. There are a particularly interesting class of static solutions called monopoles which minimise a functional (called the Yang-Mills-Higgs functional-see Eq. 2.1) subject to the condition $|\Phi| \rightarrow 1$ as $|x| \rightarrow \infty$. The space of monopoles consists of different components characterised by a topological integer $k$, which is the winding number of the Higgs field on a very large sphere. After dividing out by the action of the gauge transformations these different components are smooth manifolds of dimension $4 k-1$ and are called the moduli spaces of monopoles of degree $k$, and will be denoted $N_{k}$. As is discussed in Sects. 2-4 it is necessary for technical reasons to increase the dimension of $N_{k}$ by one to obtain a $4 k$ dimensional manifold $M_{k}$ which is a circle bundle over $N_{k}$. There is a Riemannian metric on $M_{k}$ defined by taking the $L^{2}$ inner product of tangent vectors. Properties of this metric have been discussed in detail in the book [AH88]. We will construct solutions to Eqs. 1.1-1.3 which are close to one of the monopole solutions throughout a long time period. Of course the monopole to which our solution is closest varies with time in general, and it was suggested by Manton ([Man82]) that the time evolution of this "closest monopole" should be approximately geodesic on $M_{k}$ in the slow motion limit. This paper provides a rigorous justification of this suggestion.

A similar problem for the two dimensional Abelian Higgs model has recently been discussed in [Stu]. In the present paper the formal asymptotic situation is very similar, but the proof that the asymptotics are valid is considerably more difficult. This is because in this case when the equations are linearised about a monopole the associated linear operator has a spectrum which extends all the way to the origin. This is in contrast to the case of the Abelian Higgs model, where there is a gap between the zero modes (eigenvectors with zero eigenvalue) and the rest of the spectrum. The physical origin of this different behaviour lies in symmetry breaking the reader unfamiliar with these ideas can either refer to Sect. 8.3 in [CL84] for 
physical background or ignore all the physical terminology and concentrate on the analysis. In the Abelian Higgs case there is complete symmetry breaking and all fields are massive. This means that all physical quantities decay exponentially in space and is associated with the existence of a gap in the spectrum of the linear operator which is obtained by linearising about the static solutions (see [Stu]). But in the monopole problem under discussion here the symmetry breaking is only partial-there is a residual circle symmetry, and associated with this are massless fields. Thus physical quantities do not decay exponentially-in fact the magnetic field has a Coulomb tail $\left(\sim|x|^{-2}\right)$. Associated with this is the fact that the continuous spectrum of the linear operator extends all the way to zero-see Sects. 2 and 4. We now sketch the proof given in this paper and discuss the resolution of these analytical difficulties.

Let $\left\{q_{\mu}\right\}_{\mu=0}^{4 k-1}$ be a local co-ordinate patch on $M_{k}$-this means that we have a map

$$
q \rightarrow \Psi_{0}(x ; q)
$$

which is a local diffeomorphism from an open set in $\mathbf{R}^{4 k}$ to a $4 k$ parameter family of monopoles. Such co-ordinate patches are constructed in Sect. 4, and they have the additional property that $\frac{\partial \Psi_{0}}{\partial q_{\mu}}$ is square integrable. We will produce solutions which are close to slowly varying monopoles. Slowly varying means that there is a small parameter $\varepsilon$ and the co-ordinates $q$ satisfy:

$$
\frac{d q}{d t}=O(\varepsilon) \quad \frac{d^{2} q}{d t^{2}}=O\left(\varepsilon^{2}\right) \quad \frac{d^{3} q}{d t^{3}}=O\left(\varepsilon^{3}\right)
$$

We will write $\tau=\varepsilon t$, and use the shorthand $\dot{f}=\frac{d f}{d \tau}, \ddot{f}=\frac{d^{2} f}{d \tau^{2}}$. We now make an ansatz for the solution. We use the variable $\Psi$ for $\left(A_{1}, A_{2}, A_{3}, \Phi\right)$ and search for solutions of the form:

$$
\begin{gathered}
A_{0}=O\left(\varepsilon^{3}\right), \\
\Psi(t, x)=\Psi_{0}(x ; q(t))+\varepsilon^{2} \psi(t, x),
\end{gathered}
$$

where $\psi=(\tilde{a}, \tilde{\phi})$. In order for the first condition to be consistent it is necessary to choose the gauge correctly, as we now describe.

Definition. We say that $\psi=(\tilde{a}, \tilde{\phi})=\left(\tilde{a}_{1}, \tilde{a}_{2}, \tilde{a}_{3}, \tilde{\phi}\right)$ satisfies the gauge orthogonality condition with respect to a monopole $\Psi_{0}=(a, \phi)$ if

$$
\sum_{i=1}^{3}\left(\nabla_{a}\right)_{i} \tilde{a}_{i}+[\phi, \tilde{\phi}]=0
$$

Remark. The geometric interpretation of this is that $(\tilde{a}, \tilde{\phi})$ is $L^{2}$-orthogonal to the direction of the gauge flow at $\Psi_{0}$. (Here we take the inner product on $s u(2)$ defined in Sect. 2). The gauge flow is generated by the infinitesimal gauge transformations, which are given by

$$
\begin{aligned}
& a \rightarrow-d \chi+[\chi, a], \\
& \phi \rightarrow[\chi, \phi],
\end{aligned}
$$

where $\chi$ is an $s u(2)$ valued function on $\mathbf{R}^{3}$. A simple integration by parts shows that if $(\tilde{a}, \tilde{\phi})$ satisfies the gauge orthogonality condition with respect to $\Psi_{0}$ then it is $L^{2}$-orthogonal to those infinitesimal gauge transformations generated by compactly supported $\chi$. Notice however that this is not assured if $\chi$ does not go to zero as 
$|x| \rightarrow \infty-$ in Sect. 3. This remark will be important for the case where $\chi=\phi$, the Higgs field of the monopole itself.

We will choose the gauge such that both $\dot{\Psi}_{0}$ and $\psi$ satisfy the condition of gauge orthogonality. The local existence theorem of Appendix E shows that this is possible (for small $\varepsilon$ ). This choice of gauge ensures $A_{0}=O\left(\varepsilon^{3}\right)$, and for the remainder of this introduction we will ignore $A_{0}$. We are now left with an equation for $\psi$ of the form:

$$
\frac{\partial^{2} \psi}{\partial t^{2}}+L \Psi_{0} \psi=-\ddot{\Psi}_{0}+\varepsilon J
$$

where 3 represents the nonlinear terms which are given in Appendix B, and $L \Psi_{0}$ is a second order linear differential operator. The estimates for Eq. 1.5 are for the quantity $\left(\psi, L_{\Psi_{0}} \psi\right)_{L^{2}}$, so the ideal situation would be if this quadratic form were equivalent to $|\psi|_{H^{1}}^{2}$. In fact there are three sources of degeneracy of this quadratic form:

(i) Gauge invariance gives rise to an infinite dimensional null space for $L_{\Psi_{0}}$. This is factored out by the choice of gauge just mentioned.

(ii) There is a $4 k$-dimensional space of square integrable eigenvectors $\left\{n_{\mu}\right\}_{\mu=0}^{4 k-1}$ of $L_{\Psi_{0}}$ having zero eigenvalue. These are called the zero modes and form an essential part of the problem. They arise because there are $4 k$-parameter families of monopoles. The basic idea of solitary wave perturbation theory is to choose the evolution of $q(t)$ such that

$$
\left(\psi, n_{\mu}\right)_{L^{2}}=0 \text { for } \mu=0, \ldots, 4 k-1 .
$$

Definition. We shall say that $\psi$ satisfies the dynamic condition with respect to the monopole $\Psi_{0}$ if it satisfies the conditions in Eq. 1.6 where $n_{\mu} \in \operatorname{Ker} L_{\Psi_{0}}$.

Thus if $\psi$ satisfies the dynamic condition the finite dimensional degeneracy due to the zero modes is removed. The reason for this terminology is that this condition determines the monopole dynamics, i.e. the evolution of the monopole parameters $q(t)$. This is well understood and goes back to the paper [Ben72]. This condition can be interpreted as choosing $q(t)$ such that $\Psi_{0}(x ; q(t))$ is the closest monopole to the solution at time $t$ in the $L^{2}$ sense (see [Stu]).

(iii) There is a degeneracy because the continuous spectrum reaches the origin. This is a technical obstacle which arises due to partial symmetry breaking.

Thus we use the gauge orthogonality and dynamic conditions to overcome the first two degeneracies, but on account of the third the quadratic form $\left(\psi, L \psi_{0} \psi\right)_{L^{2}}$ is definitely still not equivalent to the $H^{1}$ norm. However it is possible to prove after a very careful analysis of the linearized static problem that for smooth compactly supported sections $\psi$ satisfying the dynamic and gauge orthogonality conditions there exists a number $c$ such that

$$
c^{-1}|\psi|_{\Psi_{0}}^{2} \leqq\left(\psi, L \Psi_{0} \psi\right)_{L^{2}} \leqq c|\psi|_{\Psi_{0}^{2}}
$$

where

$$
|\psi|_{\Psi_{0}^{2}} \equiv \int\left(\left|\nabla_{a} \psi\right|^{2}+|[\phi, \psi]|^{2}\right) d x
$$

We define the space $H_{\Psi_{0}}$ to be the completion of the smooth compactly supported sections with respect to this norm. Further definitions are given in Appendix A. As 
a consequence of 1.7 we have to work with the norm $|\cdot| \Psi_{0}-$ in particular we do not have $L^{2}$ control over the component of $\psi$ in the direction of the Higgs field. This necessitates great care in the estimates. To prove that $\psi$ is bounded for times of $O\left(\frac{1}{\varepsilon}\right)$ we use the energy and higher order energy identites for Eq. 1.5 given in Sect. 6. To do this we need to estimate two types of terms:

$$
\int\left(\psi_{t}, \jmath(\psi)\right) d x \text { and } \int\left(\ddot{\Psi}_{0}, \psi\right) d x \text {. }
$$

For the first of these we need the crucial fact that (ignoring $A_{0}$ )

$$
|\mathcal{J}(\psi)|_{L_{2}} \leqq c\left(|\psi|_{\Psi_{0}}+|\psi|^{3} \Psi_{0}\right) .
$$

To explain the meaning of this in words we can say that the bad terms-the component of $\psi$ along the Higgs field, over which we have no $L^{2}$ control - is decoupled from the nonlinear evolution in an appropriate sense. This is one of the reasons that Manton's approximation is valid in spite of the radiation.

To estimate the second term we use the fact that from Sobolev's theorem

$$
|\psi|_{L^{6}} \leqq c|\psi|_{\Psi_{0}},
$$

so that if we can estimate $\ddot{\Psi}_{0}$ in $L^{6 / 5}$ we will be done. (Apply Hölder's inequality with $p=6, q=6 / 5)$. In fact on account of the dynamic condition, we only need prove that after subtracting out a linear combination of zero modes $\ddot{\Psi}_{0} \in L^{6 / 5}$. This is proved in Sect. 3 using a careful analysis of the linearized problem based on Green function estimates for the Dirac operator on Euclidean $\mathbf{R}^{3}$.

The proof of 1.7 given in Sect. 7 depends on three basic facts

(i) The quadratic form $\left(\psi, L_{\Psi_{0}} \psi\right)_{L^{2}}$ is non-negative. This is because the monopoles minimize the Yang-Mills-Higgs functional (which is defined in Sect. 2). This non-negativity can be seen explicitly in the formula 5.13, which allows us to write $L$ as a product of two first order operators whose symbol is the Dirac operator on Euclidean $\mathbf{R}^{3}$.

$$
L \Psi_{0}=\mathscr{D}_{\Psi_{0}}^{*} \mathscr{D} \Psi_{0}
$$

These operators, which are defined in Sect. 3 were studied in detail in [Tau83] and further results are given in this paper.

(ii) The following formula:

$$
L_{\Psi_{0}} u=-\Delta_{a} u-[\phi,[\phi, u]]+\frac{1}{2} \tau^{i} \varepsilon_{i j k}\left[F_{j k}, u\right]-\tau^{i}\left[\left(\nabla_{a}\right)_{i} \phi, u\right]
$$

shows that at spatial infinity the quadratic form $\left(\psi, L \Psi_{0} \psi\right)_{L^{2}}$ becomes equal to $\mid \psi_{\Psi_{0}}^{2}$.

(iii) For each zero mode $n_{\mu}$ there is a square integrable section $f_{\mu}$ such that $n_{\mu}=\mathscr{D} \Psi_{0} f_{\mu}$. This is the crucial analytical fact which combined with the standard situation given by the previous two statements allows us to prove 1.7. It is proved in Appendix A.

The dynamic condition $\left(\psi, n_{\mu}\right)_{L^{2}}=0$ leads to modulation equations for $q(t)$ which are self-contained to highest order. These are obtained by differentiating twice and substituting for $\psi_{t t}$ from Eq. 1.5. This leads to the equation:

$$
\left(\ddot{\Psi}_{0}, n_{\mu}\right)_{L^{2}}=O(\varepsilon) \text {. }
$$


If we put zero on the right-hand side this is in fact just the condition that $t \rightarrow \Psi_{0}(t)$ be a geodesic. This is because the linear span of the zero modes $n_{\mu}$ is the tangent space $T_{\Psi_{0}} M_{k}$, and our metric with respect to the co-ordinate system is given by

$$
g_{\mu \nu}=\left(n_{\mu}, n_{v}\right)_{L^{2}} .
$$

Now by our requirement that $\dot{\Psi}_{0}$ should satisfy the gauge orthogonality condition we see that $\dot{\Psi}_{0} \in T_{\Psi_{0}} M_{k}$, and the geodesic equation is just the requirement that the projection of $\ddot{\Psi}_{0}$ onto $T_{\Psi_{0}} M_{k}$ should be zero. To see this more concretely one can calculate explicitly in the co-ordinate system $q$, the condition above becomes

$$
\ddot{q}_{\mu}=-\left(g^{-1}\right)_{\mu v}\left(\left(n_{v}, \dot{n}_{\lambda}\right)_{L^{2}} \dot{q}_{\lambda}\right)+O(\varepsilon)
$$

which can be shown to be the geodesic equation by direct calculation. This calculation has been done in [Stu] and will not be repeated here.

The $O(\varepsilon)$ terms depend on $\psi$ and so our asymptotics lead to a coupled system of ordinary and partial differential equations, for which local existence is proved in Appendix E. To prove that asymptotics are valid we have to prove that for times of $O\left(\frac{1}{\varepsilon}\right),|\psi|_{L^{\infty}}$ is bounded and $q(t)$ differs from $q^{0}(t)$, the solution of the unperturbed geodesic equation with the same initial values, by $O(\varepsilon)$. We now state our main theorem using some notation defined in Appendix A:

Theorem 1.1. Consider the initial value problem for Eqs. 1.1-1.3 with initial data of the form

$$
\begin{gathered}
\Psi(0, x)=\Psi_{0}(q(0))+\varepsilon^{2} \psi(0, x), \\
\Psi_{t}(0, x)=\varepsilon \dot{q}_{\mu} n_{\mu}+\varepsilon^{2} \psi_{t}(0, x),
\end{gathered}
$$

where $\psi(0) \in L^{2},\left(\psi(0), \psi_{t}(0)\right) \in H_{3, \Psi_{0}(q(0))} \oplus H_{2, \Psi_{0}(q(0))}$ and $\psi(0)$ satisfies the $d y$ namic and gauge orthogonality conditions with respect to $\Psi_{0}(q(0))$. Then there exists $\varepsilon_{*}$ such that for $\varepsilon<\varepsilon_{*}$ there is a time $T=O\left(\frac{1}{\varepsilon}\right)$ such that there is a solution on $[0, T]$ of the form

$$
\Psi(t, x)=\Psi_{0}(q(t))+\varepsilon^{2} \psi(t, x)
$$

where $\dot{\Psi}_{0}(q(t))$ and $\psi(t)$ satisfy the gauge orthogonality with respect to $\Psi_{0}(q(t))$, and $\psi(t)$ satisfies the dynamic condition with respect to $\Psi_{0}(q(t))$. In addition $\left|A_{0}\right|_{4, \Psi_{0}(q(t))}=O\left(\varepsilon^{3}\right),|\psi(t)|_{3, \Psi_{0}(q(t))}+\left|\psi_{t}(t)\right|_{2, \Psi_{0}(q(t))}$ is bounded independent of $\varepsilon$ and

$$
\max _{[O, T]}\left|q(t)-q^{0}(\varepsilon t)\right|=O(\varepsilon)
$$

where $q^{0}(t)$ is the solution of the geodesic equation on $M_{k}$ with initial data $q(0), \dot{q}(0)$. The solution has the regularity described in Sect. 8.

This theorem leaves open two very interesting questions which are related. Firstly it is known (see [MB88]) that there are closed geodesics on $M_{k}$, so it is natural to ask whether there are corresponding periodic solutions of Eqs. 1.1-1.3, which could be interpreted as monopole bound states. Secondly it would be interesting to understand the asymptotic behaviour as $t \rightarrow \infty$ of the equations - does the solution converge in $L^{\infty}$ to an approximate superposition of monopoles as $t \rightarrow \infty$ ? 
(Something of this type occurs in the case of the $\mathrm{KdV}$ equation.) Clearly the existence of a bound state would be an obstruction to this, so if bound states do exist one might expect the asymptotic appearance of the solution to be a superposition of monopoles and bound states. Something of this type occurs for the Sine-Gordon equation, in which there are kink bound states (breathers) which together with the kinks themselves dominate the behaviour as $t \rightarrow \infty$.

Finally we summarize the contents of this paper. In Sect. 2 we review the static solutions and discuss their asymptotic decay properties. A theorem on the asymptotic appearance of monopoles at spatial infinity is proved in Appendix D. In Sect. 3 we discuss the linearised problem and obtain some results on the zero modes and their derivatives using Green function estimates for the Euclidean Dirac operator which are proved in Appendix C. In Sect. 4 we obtain local spaces of solutions by "integrating up" the zero modes with the inverse function theorem. In Sect. 5 the asymptotic expansions for monopole dynamics are developed, leading to a coupled system of equations for $A_{0}, \psi, q$ for which local existence is proved in Appendix E. In Sect. 6 some higher order energy identities are written down for the system of equations. In Sect. 7 it is shown that the higher order energies provide a good norm for the solution-equivalent to the norm used in the local existence theorem. Finally in Sect. 8 the main theorem is proved. In Appendix A various definitions and background results are collected.

The reader will find good mathematical introductions to gauge theories in [JT82] and [Ble81], while the physical background is explained in [CL84].

\section{The Monopole Solutions}

In this section we review the static situation - the equations of interest correspond to critical points of the Yang-Mills functional

$$
\mathscr{E}=\frac{1}{2} \int_{\mathbf{R}^{3}}|F|^{2}+|D \Phi|^{2} .
$$

Here all the fields are sections of vector bundles over $\mathbf{R}^{3}-F=d A+\frac{1}{2}[A, A]$ is the curvature (magnetic field) associated to a connection (vector potential) $A$ on $\mathbf{R}^{3}$ on a principal SU(2) bundle, and $\Phi$ is a section of an associated $s u(2)$ bundle. Since the base space is $\mathbf{R}^{3}$ these bundles are all trivial and so the fields are just $s u(2)$ valued functions. The Lie group SU(2) is the group of unitary two by two matrices with determinant equal to +1 . The Lie algebra $s u(2)$ is the vector space of traceless, skew-hermitian two by two matrices with the multiplication given by commutation:

$$
[A, B]=A B-B A .
$$

For an inner product on $s u(2)$ we take

$$
A \cdot B=(A, B)=-\frac{1}{2} \operatorname{tr}(A B) .
$$

This inner product has the following useful invariance property:

$$
([A, B], C)+(B,[A, C])=0 .
$$

We can then take as orthonormal basis 


$$
e_{1}=\left(\begin{array}{cc}
i & 0 \\
0 & -i
\end{array}\right) e_{2}=\left(\begin{array}{cc}
0 & 1 \\
-1 & 0
\end{array}\right) e_{3}=\left(\begin{array}{ll}
0 & i \\
i & 0
\end{array}\right)
$$

We can now see that $s u(2)$ is isomorphic to $\mathbf{R}^{3}$ with the vector product giving the Lie bracket operation since

$$
\left[e_{1}, e_{2}\right]=2 e_{3} \text {. }
$$

The covariant derivative of an $s u(2)$-valued function is $D_{A} f=d f+[A, f]$. The subscript $A$ will be omitted whenever no confusion is possible.

The functional defined in Eq. 2.1 is invariant under the gauge transformations. These are given as follows - if $g(x)$ is a differentiable SU(2)-valued function, then it generates a gauge transformation according to:

$$
A \rightarrow g \partial g^{-1}+g A g^{-1} \quad \Phi \rightarrow g \Phi g^{-1} \quad F \rightarrow g F g^{-1},
$$

which will be written as

$$
(A, \Phi) \rightarrow \rho(g)(A, \Phi) .
$$

The multi-monopoles appear as minima of the functional defined in Eq. 2.1 within a given topological sector. The different topological sectors are specified by requiring that

$$
|\Phi| \rightarrow 1 \text { as }|x| \rightarrow \infty .
$$

Then at spatial infinity $\Phi$ defines a map between spheres whose degree $k$ is the required topological number. To be more precise in [Gro84] it is proved that if the following conditions are satisfied:

(i) $\lim _{R \rightarrow \infty} \sup _{|x|=R}(1-|\Phi|)=0$,

(ii) $\mathscr{E}(A, \Phi)<\infty$,

then

$$
\frac{1}{2 \pi} \operatorname{tr} \int D \Phi \wedge F=\lim _{r \rightarrow \infty} \frac{1}{4 \pi} \int_{|x|=R}|\Phi|^{-1}(\Phi, F)=k_{1} \in \mathbf{Z} .
$$

We now minimise $\mathscr{E}$ in the space such that $A, \Phi$ and their first derivatives are locally square integrable. Now from Sect. 4.11 of [JT82] it is known that for finite action critical points of $\mathscr{E}$ there exists a number $M$ such that $\lim _{R \rightarrow \infty} \sup _{|x|=R}(M-|\Phi|)=$ 0 . We can then rescale to make $M=1$ (see p. 102 of [JT82]). Thus we lose nothing by restricting further the space on which we minimise by requiring the condition (i) above to hold. To see the importance of this we use formula 2.7 to write a Bogomolny decomposition:

$$
\mathscr{E}=\frac{1}{2} \int_{\mathbf{R}^{3}}\left|{ }^{*} F \mp D \Phi\right|^{2} \pm 4 \pi k_{1}
$$

The minima of $\mathscr{E}$ with negative winding number will therefore be solutions of the Bogomolny equations

$$
{ }^{*} F=-D \Phi \text { or } \quad F_{l n}=\varepsilon_{l m n} D_{m} \Phi .
$$

It will in fact follow from the decay results in Eqs. 2.12-2.14 that the integer $k_{1}$ is equal to the winding number of $\Phi$ on large spheres (see p.44 in [JT82]). The minima will also be solutions of the Euler-Lagrange equations corresponding to the functional in Eq. 2.1: 


$$
\begin{aligned}
-\sum_{l=1}^{3} D_{l}^{2} A_{i}+D_{l} \partial_{i} A_{l} & =-\left[\Phi, D_{i} \Phi\right], \\
-\sum_{l=1}^{3} D_{l}^{2} \Phi & =0 .
\end{aligned}
$$

The Bogomolny equations can be studied both by analytical methods (see [JT82, Tau82]) and by integrable systems methods (see [Hit82, Hit83, AH88, Don84, ES89]). From analytical considerations it is known that all solutions are smooth in an appropriate gauge - in fact any solution of the second order Euler-Lagrange equations which has the property that there exists a gauge in which $A, \Phi$ and their first derivatives are locally square integrable is gauge equivalent to a smooth solution and locally gauge equivalent to a real analytic one. In addition we have the following decay properties for our monopoles:

$$
\begin{aligned}
& \text { (i) }|D \phi|=\frac{k}{|x|^{2}}+O\left(|x|^{-3+\varepsilon)},\right. \\
& \text { (ii) }|\phi|=1-\frac{k}{2|x|}+O\left(|x|^{-2}\right), \\
& \text { (iii) } \frac{\partial|\phi|}{\partial \Omega}=O\left(|x|^{-2}\right)
\end{aligned}
$$

where $\frac{\partial}{\partial \Omega}$ means angular derivative, and $\varepsilon \in(0,1)$. This follows from the results of [JT82], and is proved in Appendix D. This provides a link with the integrable systems methods where the solutions of the Bogomolny equations satisfying these decay properties are studied and parametrised using algebraic geometry. Since all the minima satisfy these decay properties there is a full equivalence between the classes of solutions studied with analysis and with integrable systems methods.

It is known that the moduli space of solutions (that is the space of gauge equivalence classes of solutions) for the Bogomolny equations with these boundary conditions is a $4 k-1$ dimensional smooth manifold, which we shall call $N_{k}$. For example if $k=1$ there are three parameters corresponding to the centre of the monopole. If the centre is chosen at the origin the solution can be written explicitly:

$$
\begin{aligned}
\phi & =-\left(\frac{1}{\tanh r}-\frac{1}{r}\right) \mathbf{n} \cdot \mathbf{e}, \\
a(x) & =\left(\frac{1}{\sinh r}-\frac{1}{r}\right) \mathbf{n} \times \mathbf{e} \cdot d \mathbf{x},
\end{aligned}
$$

a solution known as the Prasad-Sommerfeld solution, which is known to be unique among the radially symmetric solutions ([Mai81]). Here $\mathbf{n}=\mathbf{x} /|\mathbf{x}|$ and $\mathbf{e}$ is the orthonormal basis for $s u(2)$ defined in Eq. 2.4. We shall be concerned with these $4 k-1$ parameter families of solutions, which we will generically write as the gauge equivalence classes

$$
\Psi_{0}(x ; q) \equiv(a(x ; q), \phi(x ; q)) \sim \rho(g) \Psi_{0}(x ; q)=\left(g d g^{-1}+g a(x ; q) g^{-1}, g \phi(x ; q) g^{-1}\right)
$$

for a set of local parameters $\left\{q_{\mu}\right\}_{\mu=1}^{4 k-1}$ on the moduli space. The existence of such families of solutions was first suggested by an informal parameter counting of 
Weinberg ([Wei79]) and the construction of $3 \mathrm{k}$ parameter families by patching of Prasad Sommerfeld monopoles sufficiently well separated ([JT82]). Later in [Tau83] a rigorous index calculation was given to confirm the number $4 k-1$ (subject to a proviso on an additional "non-physical" zero mode discussed below). Integrable systems methods confirmed this and gave much additional information ([Hit82, Hit83, Don84, ES89]) - in particular there is a circle bundle $M_{k}$ over $N_{k}$, whose definition will be reviewed below, which was shown in [Don84] to be identical with the space of base point preserving rational maps. The solutions can be intepreted as approximate nonlinear superpositions of Prasad-Sommerfeld monopoles in the limit of large separation, and for this reason the solutions are often referred to as multi-monopoles for $|k|>1$.

In [JT82] there are some further estimates for these solutions which we should keep in mind. The reader will have observed from Eqs. 2.12-2.14 that the solutions approach their asymptotic values algebraically. This is in distinction to the situation in the Abelian Higgs model where all physical quantities - for example the magnetic field and the energy density - decay exponentially (see [JT82, Stu]). In physical terminology the reason for this is that for the multi-monopoles to exist it is necessary that there be only partial symmetry breaking (see [CL84]). This is because the magnetic field from a monopole in three dimensions has a Coulomb tail $\left(\sim|x|^{-2}\right)$ so if multi-monopoles are to exist there must be an attractive force to cancel out the Coulomb repulsion. This is provided by the Higgs field, which must therefore also approach its asymptotic value algebraically. We now make some informal comments which will hopefully make this clearer - for details the reader should refer to [JT82]. Mathematically, if there is to be exponential decay the linearised equations at spatial infinity must look schematically like

$$
\left(-\Delta+m^{2}\right) u=0 \text {. }
$$

If this is the case then $m$ is called the mass and controls the exponential decay rate (see [JT82, Chapter three]). This is how things work out for the Abelian Higgs model, and the asymptotic value of $|\Phi|$ determines the mass. However this cannot and does not occur in the monopole case for the reasons just mentioned-there are still massless directions in which the decay is algebraic. If we linearise the equations at spatial infinity then we end up with equations which look schematically like

$$
-\Delta u-[\phi,[\phi, u]]=0,
$$

where $u$ is an $s u(2)$ valued quantity. Thus there will only be exponential decay in the directions which are transverse to the Higgs field. Consequently in order to state decay results we have to decompose the $s u(2)$ valued quantities $\chi$ as

$$
\chi=\chi_{L}+\chi_{T} \quad \chi_{L}=(\chi, \phi) \phi /\left(|\phi|^{2}\right)
$$

into longitudinal and transverse components relative to the Higgs field. We can now state the decay results which are proved in [JT82, Chapter Four]. In the transverse (massive) directions we have exponential decay (broken symmetry):

$$
\left|(D \phi)_{T}\right|,\left|F_{T}\right| \leqq c(\varepsilon) e^{-(1-\varepsilon)|x|}
$$

while in the longitudinal (massless) directions there is slow algebraic decay (unbroken symmetry):

$$
0 \leqq(1-|\phi|) \leqq c /|x| \quad\left|(D \phi)_{L}\right| \leqq c /\left(|x|^{2}\right) .
$$


It was mentioned in the introduction that this is related to behaviour of the Hessian of $\mathscr{E}$, which we will call $\operatorname{Hess} \Psi_{0}$ and we now explain how this comes about. To understand the Hessian properly it is first of all necessary to "correct" for gauge invariance. So to look at the value of the Hessian in the direction of the variation $\psi=(\tilde{a}, \tilde{\phi})$ we will require $\psi$ to satisfy the gauge orthogonality condition with respect to the background monopole $\psi_{0}$, and therefore we can introduce a modified Hessian

$$
\overline{\operatorname{Hess}}_{\Psi_{0}}(\psi, \psi)=\operatorname{Hess} \Psi_{0}(\psi, \psi)+\int\left(\sum_{i=1}^{3}\left(\nabla_{a}\right)_{i} \tilde{a}_{i}+[\phi, \tilde{\phi}]\right)^{2} d x,
$$

which takes on exactly the same value as the Hessian on gauge orthogonal directions $\psi$. A direct calculation then shows that

$$
\overline{\operatorname{Hess}} \Psi_{0}(\psi, \psi)=\int\left(\left|\nabla_{a} \psi\right|^{2}+|[\phi, \psi]|^{2}+\left(\psi, \frac{1}{2} \tau^{i} \varepsilon_{i j k}\left[F_{j k}, u\right]-\tau^{i}\left[\left(\nabla_{a}\right)_{i \phi}, u\right]\right) .\right.
$$

As usual the critical points of the Hessian give the linearised equations and indeed one can check that

$$
\overline{\operatorname{Hess}} \Psi_{0}(\psi, \psi)=\left(\psi, L_{\Psi_{0}} \psi\right)_{L^{2}} \text {. }
$$

This explains the relation between the appearance of the linearised equations and the degeneracy of the Hessian. This formula for $\overline{\text { Hess }}$ illustrates two important points firstly the gauge orthogonality condition ensures that all derivatives of $\psi$ are contained in modified Hessian (whereas for general variations $\psi$ only the exterior derivatives appear in the ordinary Hessian). Secondly as $|x| \rightarrow \infty$ the modified Hessian approaches the norm $|\psi|_{\Psi_{0}}$ defined in the introduction which is crucial to proving that they are equivalent norms on the subspace orthogonal to the zero modes - see Sect. 7. Thus we see that we get $L^{2}$ control only over the massive fields, i.e. those which decay exponentially. The existence of massless fields has as a consequence the degeneracy of $\overline{\text { Hess }}$.

\section{The Zero Modes}

The zero modes are the solutions of the linearised Bogomolny equations which satisfy the condition of gauge orthogonality defined in Eq. 1.4. These can be written in an economical way by introducing the quaternionic notation as in [Tau83]. So let $\left\{\tau^{i}\right\}_{i=1}^{3}$ satisfy

$$
\tau^{i} \tau^{j}=-\delta^{i j}+\varepsilon^{i j k} \tau^{k}
$$

where $\delta$ is the Kronecker delta symbol and $\varepsilon^{i j k}$ is the completely antisymmetric tensor. These are the purely imaginary quaternions - the whole quaternion algebra is generated by these together with 1 . In this section we want to linearise the Bogomolny equations so we consider

$$
\Psi=(A, \Phi)=\Psi_{0}+\psi=(a, \phi)+(\tilde{a}, \tilde{\phi}),
$$

where $\Psi_{0}=(a, \phi)$ is a monopole. Using the quaternionic notation we can write $\psi=(\tilde{a}, \tilde{\phi})$ as

$$
\psi=\sum_{i=1}^{3} \tau^{i} \tilde{a}_{i}+\tilde{\phi}
$$


Notation. A summation convention will often be used for repeated indices. The $s u(2)$ inner product together with the standard inner product on $\mathbf{H}=\mathbf{R}^{4}$ gives an inner product $(\cdot, \cdot)$ on $s u(2) \otimes \mathbf{H}$. We will use $\phi \cdot n$ to denote the quaternion obtained by taking the $s u(2)$ inner product of $\phi \in s u(2)$ and $n \in s u(2) \otimes \mathbf{H}$.

A calculation shows that the linearised Bogomolny equations together with the condition of gauge orthogonality for $\psi$ with respect to $\Psi_{0}$ can be written as the single quaternionic equation

$$
\mathscr{D}_{\Psi_{0}} \psi=0 \text {, where } \mathscr{D}_{\Psi_{0}}=-\tau^{i}\left(\nabla_{a}\right)_{i}+[\phi, \cdot] \text {. }
$$

This operator was studied in [Tau83], where it is shown that it is a Fredholm operator from $H_{\Psi_{0}}$ to $L^{2}$, where these spaces are defined to be the completions of smooth, compactly supported sections of the $s u(2) \otimes \mathbf{H}$ bundle with respect to the norms

$$
\begin{aligned}
|\psi|_{\Psi_{0}^{2}} & =\left|\nabla_{a} \psi\right|_{L^{2}}^{2}+|[\phi, \psi]|_{L^{2}}^{2}, \\
|\psi|_{L^{2}} & =\int \sum_{\mathbf{R}^{3}}^{3}\left|\tilde{a}_{i}\right|^{2}+|\tilde{\phi}|^{2} d x .
\end{aligned}
$$

Theorem 3.1 (Taubes). Let $\Psi_{0}=(a, \phi)$ represent a $k$-monopole, then there are exactly $4 k$ solutions of $\mathscr{D}_{\Psi_{0}} \psi=0$ lying in $H_{\Psi_{0}}$. These are called zero modes and will be denoted $\left.n_{\mu}\right|_{\mu=0} ^{4 k-1}$, while $T_{\Psi_{0}} M_{k}$ will be used to denote their linear span.

Remark. Notice that the quaternions act on $T_{\Psi_{0}} M_{k}$ by right multiplication.

Remark. Of these $4 k$ zero modes, $4 k-1$ should be regarded as corresponding to the physical parameters of the moduli space. The remaining zero mode refers to an infinitesimal gauge transformation produced by the Higgs field itself:

$$
n_{0} \equiv \sum_{i=1}^{3} \tau^{i}\left(\nabla_{a}\right)_{i} \phi
$$

This is not excluded by the condition of gauge orthogonality - in fact since the Higgs field itself does not decay at infinity the derivation of the gauge orthogonality condition does not apply to the case where the gauge transformation is the Higgs field itself. The existence of such a "non-physical" zero mode is related to the fact that the symmetry breaking is partial - there are still massless directions longitudinal relative to the Higgs field. For example in the two dimensional Abelian Higgs model the symmetry breaking is complete and there are no such "non-physical" zero modes appear. Thus the true number of parameters which the monopoles depend on is $4 k-1$, which agrees with the integrable systems parameter counting (see [Hit82, Hit83]). To deal with the extra zero mode it is convenient to consider instead of the moduli space $N_{k}$ a $4 k$-dimensional space which is a circle bundle over $M_{k}$, with the gauge transformations induced by the Higgs field itself defining the circle action. This then gives a correct match between the dimension of the space and the number of zero modes. The definition of $M_{k}$ discussed in detail in the succeeding sections.

We are interested in curves $\tau \rightarrow \Psi_{0}(\tau) \in M_{k}$ whose velocity vectors at the point $\Psi_{0}(\tau)$ lie in $T_{\Psi_{0}} M_{k}$. If $\Psi_{0}(\tau)=\tau^{i} a_{i}(x ; q(\tau))+\varphi(x ; q(\tau))$ then this amounts to requiring that 


$$
\frac{d}{d \tau}\left(\tau^{i} a_{i}+\varphi\right)=\sum_{\mu=0}^{4 k-1} \dot{q}_{\mu} n_{\mu},
$$

where $n_{\mu} \in T_{\Psi_{0(\tau)}} M_{k}$. We will show in the next section that given a curve in $M_{k}$ we can apply a gauge transformation to ensure that the velocity vector at each point satisfies this condition. We will need the following basic facts about the first two time derivatives of the zero modes:

$$
\dot{n}_{\mu}=\frac{d}{d \tau}\left(n_{\mu}(x ; \tau) \text { and } \ddot{n}_{\mu}=\frac{d^{2}}{d \tau^{2}}\left(n_{\mu}(x ; \tau)\right.\right.
$$

which are known a priori to be in $H_{\Psi_{0}}$.

Lemma 3.2. $|n|$ and $\left|\nabla_{a} n\right|$ and their $\tau$ derivatives decay uniformly to zero, i.e.

$$
\lim _{R \rightarrow \infty|x|=R} \sup _{R}\left(|n|+\left|\nabla_{a} n\right|+|\dot{n}|+\left|\nabla_{a} \dot{n}\right|+|\ddot{n}|+\left|\nabla_{a} \ddot{n}\right|\right)=0 \text {. }
$$

Proof. Part of the proof follows very closely arguments in [JT82] and the reader may want to have that book in hand. In view of Lemma A.5 we only need prove that $|n|, \nabla|n|, \nabla_{i} \nabla_{j}|n|$ are in $L^{6}$. To see that this is so we apply Theorem A.7 to the identity

$$
-\left(\nabla_{a}\right)^{2} \nabla_{j} n=2\left[F_{j i},\left(\nabla_{a}\right)_{i} n\right]+\left[\left(\nabla_{a}\right)_{i} F_{j i}, n\right]+\tau^{i}\left[\varepsilon^{i j k} F_{j k}+\left(\nabla_{a}\right)_{i \phi}, n\right]+[\phi,[\phi, n]] .
$$

Notation. In regions where $|\phi(x)|>0$ we define the longitudinal and transverse components of an $s u(2)$ valued function by

$$
f^{L}=|\phi|^{-2}(f, \phi) f^{T}=f-f^{L} .
$$

Since we know that the magnitude of the Higgs field of a monopole configuration approaches 1 uniformly as $|x| \rightarrow \infty$ we can state decay results with regard to this decomposition.

Lemma 3.3. Let $n \in T_{\Psi_{0}} M_{k}$ then for any $\delta \in(0,1)$ there exist numbers $M\left(\delta,|n|_{\Psi_{0}}\right)$, $M\left(\mid n \Psi_{0}\right)$ such that

$$
\begin{aligned}
& \left|n^{T}(x)\right| \leqq M\left(\delta,|n| \Psi_{0}\right) e^{-(1-\partial)|x|} \\
& \left|n^{L}(x)\right| \leqq \frac{M\left(|n| \Psi_{0}\right)}{(1+|x|)^{2}}
\end{aligned}
$$

Remark. Notice that a priori there is no reason to suppose that the zero modes are square integrable. This lemma implies that this is the case. The proof given here depends on results for the Dirac operator $\tau^{j} \partial_{j}$ given in Appendix C. The fact that all the zero modes are square integrable is proved by a different method in [Tau83].

Proof. The first statement will be proved by the methods of chapter four in [JT82], see in particular pages 164-166. Thus the crucial point is the following identity for $\eta=[\phi, n]:$ 


$$
\begin{aligned}
-\Delta|\eta|^{2} & +2|\phi|^{2}|\eta|^{2}+4\left(\eta,\left[\nabla_{a \phi}, \nabla_{a} n\right]\right)+2\left|\nabla_{a} \eta\right|^{2} \\
& +\left(\eta,\left[\phi,\left[\tau^{i}\left(\varepsilon^{i j k} F_{j k}-\left(\nabla_{a}\right)_{i \phi}\right), n\right]\right]\right)=0 .
\end{aligned}
$$

Claim. For any $\mu \in(0,1)$ there is a function $v_{v}$, which goes to zero uniformly as $|x| \rightarrow \infty$, such that

$$
-\Delta|\eta|^{2}+2(1-\mu)\left|\nabla_{a} \eta\right|^{2} \leqq-2|\eta|^{2}(1-v(\mu))+w|\eta|,
$$

where $w$ decays exponentially:

$$
|w(x)| \leqq c(\delta) e^{-(1-\delta)|x|} \text { for any } \delta>0 .
$$

Proof of Claim. We have to estimate the terms in the previous identity. First of all notice that since $\phi \cdot \eta=0$ we can write:

$$
\left(\eta,\left[\nabla_{a} \phi, \nabla_{a} n\right]\right)=\left(\eta,\left[\left(\nabla_{a} \phi\right)^{T},\left(\nabla_{a} n\right)^{L}\right]\right)+\left(\eta,\left[\left(\nabla_{a \phi}\right)^{L},\left(\nabla_{a} n\right)^{T}\right]\right) .
$$

Using the exponential decay of $\left(\nabla_{a} \phi\right)^{T}$ the first of these is easily estimated in the desired fashion. For the second we use the following vector product identity (see p.154 of [JT82]):

$$
\begin{aligned}
|\phi|^{2}\left(\left(\nabla_{a}\right)_{j} n\right)^{T} & =\left[\phi,\left[\left(\nabla_{a}\right)_{j} \phi, n\right]\right]-\left[\phi,\left(\nabla_{a}\right)_{j} \eta\right] \\
& =\left[\left[\phi,\left(\nabla_{a}\right)_{j} \phi\right], n\right]+\left[\left(\nabla_{a}\right)_{j} \phi,[\phi, n]\right]-\left[\phi,\left(\nabla_{a}\right)_{j} \eta\right] .
\end{aligned}
$$

Again using the exponential decay of $\left[\phi,\left(\nabla_{a}\right)_{j} \phi\right]$ we can estimate these as required. In a similar way we write

$$
\begin{aligned}
\left(\eta,\left[\phi,\left[\tau^{i}\left(\varepsilon^{i j k} F_{j k}-\left(\nabla_{a}\right)_{i} \phi, n\right]\right]\right)=\right. & \left.\left.\left(\eta,\left[\phi, \tau^{i}\left(\varepsilon^{i j k} F_{j k}-\left(\nabla_{a}\right)_{i} \phi\right)\right], n\right]\right]\right) \\
& \left.+\left(\eta,\left[\tau^{i}\left(\varepsilon^{i j k} F_{j k}-\left(\nabla_{a}\right)_{i \phi}\right), \eta\right]\right]\right) .
\end{aligned}
$$

Using the exponential decay of the transverse fields we estimate these terms as required, completing the proof of the claim.

Now to prove the first statement of the lemma we proceed as on p. 167 of [JT82] with a very minor modification to deal with the extra exponentially decaying term. Let $\mu=\varepsilon / 2$ and let $R(\varepsilon)$ be sufficiently large that

$$
\sup _{|x|>R(\varepsilon)}|v(\varepsilon / 2, x)|<\varepsilon / 2,
$$

which is possible on account of the foregoing result on uniform decay. Now choose a number

$$
M(\varepsilon)>e^{(1-\varepsilon) R(\varepsilon)} \sup _{|x| \leqq R(\varepsilon)}|\eta(x)|
$$

and define a set

$$
V=\left\{x:|\eta(x)|>M(\varepsilon) e^{-(1-\varepsilon)|x|}\right\} .
$$

Then we can find a slightly larger set $V^{\prime}$ on which $|\eta|$ is strictly positive, with a smooth boundary, such that

$$
|\eta(x)| \leqq M(\varepsilon) e^{-(1-\varepsilon)|x|} \text { for all } x \in V^{\prime} .
$$


Now our aim is to apply Lemma A.6 so we have to find some way to eliminate the troublesome $\left|\nabla_{\eta}\right|^{2}$ term. To do this we consider $|\eta|^{1+\varepsilon / 2}$ (following [JT82]). This satisfies:

$$
\begin{aligned}
\Delta|\eta|^{1+\varepsilon / 2}= & \frac{1}{2}\left(1+\frac{\varepsilon}{2}\right)|\eta|^{\varepsilon / 2-1} \Delta|\eta|^{2}-\left.\left(1-\frac{\varepsilon^{2}}{4}\right)|\eta|^{\varepsilon / 2-1}|\nabla| \eta\right|^{2} \\
\geqq & \left(1-\frac{\varepsilon^{2}}{4}\right)|\eta|^{\varepsilon / 2-1}\left(\left|\nabla_{a} \eta\right|^{2}-\left.|\nabla| \eta\right|^{2}\right)+|\eta|^{\varepsilon / 2+1}\left(1+\frac{\varepsilon}{2}\right)(1-v) \\
& +\frac{1}{2}\left(1+\frac{\varepsilon}{2}\right)|\eta|^{\frac{\varepsilon}{2}} w .
\end{aligned}
$$

We now use Kato's inequality and $|\eta|^{\varepsilon / 2} \leqq 1+|\eta|^{1+\varepsilon / 2}$ to derive the differential inequality

$$
-\Delta|\eta|^{1+\varepsilon / 2} \leqq-\left(1+\frac{\varepsilon}{2}\right)\left(1-v-\frac{|w|}{2}\right)|\eta|^{1+\varepsilon / 2}+\frac{1}{2}(1+\varepsilon / 2)|w| .
$$

We can now apply Lemma A.6 to obtain the result.

To prove the second statement of the lemma we consider the Dirac operator $\tau^{j} \partial_{j}$, and notice that by the invariance of the inner product (Eq. 2.3)

$$
\begin{aligned}
\tau^{j} \partial_{j}(\phi \cdot n) & =\left(\tau^{j}\left(\nabla_{a}\right)_{j} \phi \cdot n\right)+\phi \cdot\left(\tau^{j}\left(\nabla_{a}\right)_{j} n-[\phi, n]\right) \\
& =\tau^{j}\left(\left(\nabla_{a}\right)_{j} \phi \cdot n\right) \text { since } \mathscr{D}_{\Psi_{0}} n=0 .
\end{aligned}
$$

We can now use the Green function for $\tau^{j} \partial_{j}$ to learn things about $\phi \cdot n$. The necessary results are given in Lemma C.1. We first apply case A using the known decay of $D \phi$ together with the fact that $n \in L^{6}$ from the Sobolev inequality. We then substitute the result of this and apply Case B to deduce the second statement of the lemma.

\section{Corollary 3.4}

$$
\operatorname{ker} \mathscr{D} \Psi_{0} \cap H_{\Psi_{0}}=\operatorname{Ker} \mathscr{D} \Psi_{0} \cap L^{2} .
$$

It turns out that it is absolutely essential for our method of proof that the time derivatives of $n \equiv \dot{\Psi}_{0}=(\dot{a}, \dot{\phi})$ should, modulo $T_{\Psi_{0}} M_{k}$, decay more rapidly than $n$ itself. We now set about proving this.

Lemma 3.5. It is possible to decompose

$$
\dot{n}=m+\sum c_{\mu} n_{\mu} \quad \ddot{n}=l+\sum d_{\mu} n_{\mu}
$$

in such a way that for any positive number $\delta$ there exist constants such that

$$
|m \cdot \phi| \leqq \frac{c(\delta)}{(1+|x|)^{3-\delta}} \quad|l \cdot \phi| \leqq \frac{c(\delta)}{(1+|x|)^{3-\delta}} .
$$

The numbers $c(\delta)$ depend only on $\delta,|n|_{\Psi_{0}},|\dot{n}| \Psi_{0},|\ddot{n}| \Psi_{0},\left|(1+|x|)^{2} \nabla_{a} \phi\right|_{L^{\infty}}$.

Proof. The proof uses the Green function estimates of Lemma C.1, but this time we have to take advantage of the fact that it is possible to choose $c_{\mu}, d_{\mu}$ in such a way that the integral of the right-hand side is zero. To start with notice that 


$$
\mathscr{D} m=\mathscr{D} \dot{n}=-\tau^{j}\left[\dot{a}_{j}, n\right]+[\dot{\phi}, n]
$$

so

$$
\begin{aligned}
\tau^{j} \partial_{j}(\phi \cdot m)= & \phi \cdot \mathscr{D} \dot{n}+\tau^{j}\left(\nabla_{a}\right)_{j} \phi \cdot m \\
= & -\tau^{j}\left(\phi,\left[\dot{a}_{j}, n\right]+[\dot{\phi}, n]\right)+\tau^{j}\left(\left(\nabla_{a}\right)_{j} \phi, \dot{n}\right) \\
& -\sum c_{\mu} \tau^{j}\left(\left(\nabla_{a}\right)_{j} \phi, n_{\mu}\right) .
\end{aligned}
$$

Claim. It is possible to choose the $c_{\mu}$ in such a way that the integral of the righthand side is zero.

Proof of Claim. To see this we only need show that the quaternions

$$
\tau^{j} \int\left(\nabla_{a}\right)_{j} \phi \cdot n_{\mu} \quad \mu=1, \ldots 4 k
$$

span the whole of $\mathbf{H}$. To see that this is so first of all notice that one of the zero modes is $n_{1}=\tau^{j}\left(\nabla_{a}\right)_{j} \phi$ and for this the above expression is real:

$$
\tau^{j} \tau^{k} \int\left(\left(\nabla_{a}\right)_{j} \phi \cdot\left(\nabla_{a}\right)_{k} \phi\right)=-\int\left|\nabla_{a} \phi\right|^{2} .
$$

Now recall that the quaternions act on the space of zero modes by right multiplication $n \rightarrow n q q \in \mathbf{H}$. Thus the claim is clearly true.

Now to prove the lemma we can appeal to Case C of Lemma C.1. Notice that this is a version of well known results for solutions of Poisson's equation (see [JT82, Chapter 6]) adapted for the Dirac operator $\tau^{j} \partial_{j}$. To see what the numbers $c(\delta)$ depend on notice that the coefficients $c_{\mu}$ are bounded in terms of the stated quantities. Finally we can apply an identical argument for $\ddot{n}$.

Remark. It will turn out that the crucial thing is that $\dot{n}, \ddot{n}$ are in $L^{6 / 5}$ modulo $T_{\Psi_{0}} M_{k}-$ we shall write $\Pi \dot{n}$ for this rapidly decaying component.

\section{Local Spaces of Solutions}

As we have noted it is known that $M_{k}$ is a smooth $4 k$-dimensional manifold with a complete Riemannian metric and a hyperkahler structure whose tangent space $T_{\Psi_{0}} M_{k}$ at the monopole $\Psi_{0}$ can be identified with the zero modes for the operator $\mathscr{D}_{\Psi_{0}}$ (see [AH88]). In this section we produce local families of monopoles in a form suitable for our work by integrating up the zero modes discussed in the previous section. let $\Psi_{0}=(a, \phi)$ be some fixed monopole, then we search for nearby monopoles $\Psi_{0}+\psi$, where $\psi=(\tilde{a}, \tilde{\phi})$ satisfies the deformation equation:

$$
\mathscr{D} \Psi_{0} \psi=g(\psi)
$$

with

$$
g(\psi) \equiv \tau^{k}\left(\frac{1}{2} \varepsilon_{i j k}\left[\tilde{a}_{i}, \tilde{a}_{j}\right]+\left[\tilde{a}_{k}, \tilde{\phi}\right]\right)
$$

For brevity we will omit the subscript on $\mathscr{D}$. We will first of all show that the $4 k$ zero modes integrate up to a local family of monopoles depending on $4 k$ parameters. Then we will look at some decay properties in greater detail. 
Consider the space

$$
l=\left\{v \in H_{\Psi_{0}}:(v, n)_{\Psi_{0}}=0 \quad \forall n \in T_{\Psi_{0}} M_{k}\right\} .
$$

On this space we know that the quadratic form $|\mathscr{D} \psi|$ is equivalent to the norm $|\psi| \Psi_{0}$. Therefore by the Riesz representation theorem if $\mathscr{D}^{*} g$ defines, via the $L^{2}$-pairing, a bounded linear functional on $l$ then we will obtain a solution to the equation

$$
\mathscr{D}^{*} \mathscr{D} \psi=\mathscr{D}^{*}(g),
$$

$g$ being assumed known, since the inhomogeneous term automatically satisfies the Fredholm conditions needed to apply Theorem A.9. To see that the right-hand side is a bounded linear functional on $l$ we note that

$$
\begin{aligned}
\left|\int\left(u, \mathscr{D}^{*}(g(\psi))\right)\right| & =\left|\int(\mathscr{D} u, g(\psi))\right| \\
& \leqq c|u|_{\Psi_{0}}|\psi|_{\Psi_{0}}^{2}
\end{aligned}
$$

from Lemma A.4. Let us write the solution of this equation as $G \mathscr{D}^{*}(g(\psi))$, so we can consider the map

$$
\begin{aligned}
F: H_{\Psi_{0}} & \rightarrow H_{\Psi_{0}} \\
\psi & \mapsto \psi-G \mathscr{D}^{*}(g(\psi)) .
\end{aligned}
$$

This maps solutions of the deformation equation to $T_{\Psi_{0}} M_{k}=\operatorname{Ker} \mathscr{D}$, and has derivative equal to the identity at the origin. It is therefore a local diffeomorphism and produces a local $4 k$ parameter family of solutions of the deformation equation, with $|\psi|_{\Psi_{0}}$ small. As we will discuss later in this section all but one of these are genuine monopole parameters, while the remaining parameter corresponds to the one parameter family of gauge equivalent solutions produced by the action of the Higgs field as a gauge transformation.

We need some more detailed information on the behaviour of these local solutions at spatial infinity. First of all we notice that by differentiating the deformation equation once that the second derivatives of $\psi$ are square integrable. Therefore by the Sobolev lemma the first derivatives are in $L^{6}$ and so we can apply Lemma A.5 to deduce that

$$
\lim _{R \rightarrow \infty} \sup _{|x|=R}|\psi(x)|=0
$$

We will now improve this:

Claim $A$. The solution $\psi$ of the deformation equation just obtained has the following decay properties:

$$
\begin{aligned}
& |[\phi, \psi]| \leqq c(\varepsilon) e^{-(1-\varepsilon)}|x| \\
& |\phi \cdot \psi| \leqq c(1+|x|)^{-2}
\end{aligned}
$$

Proof of Claim. First we will prove the first statement. Let $\eta=[\phi, \psi]$, then as in the previous section we obtain the following identity:

$$
\begin{aligned}
-\Delta|\eta|^{2} & +2|\phi|^{2}|\eta|^{2}+4\left(\eta,\left[\nabla_{a} \phi, \nabla_{a} n\right]\right)+2\left|\nabla_{a} \eta\right|^{2} \\
& +\left(\eta,\left[\phi,\left[\tau^{i} \varepsilon^{i j k} F_{j k}-\left(\nabla_{a}\right)_{i} \phi, n\right]\right]\right)+\left(\eta,\left[\phi, \mathscr{D}^{*} g\right]\right)=0 .
\end{aligned}
$$


Now the important thing about the last term on the right-hand side is that $g$ is a quadratic commutator so that the longitudinal terms commute out and so the expression is quadratic with respect to $\eta, \nabla \eta$. Furthermore assume that we are in a gauge such that $a \rightarrow 0$ as $|x| \rightarrow \infty$ then we can write this final expression as

$$
\alpha(x)(\eta, \eta)+\beta(x)(\eta, \nabla, \eta),
$$

where $\alpha, \beta$ are bilinear forms whose coefficents decrease to zero as $|x| \rightarrow \infty$ by Eq. 4.2. But this puts us in exactly the same situation as in the proof of Lemma 3.3 and the result is proved. To prove the second statement we use the formula:

$$
\begin{aligned}
\tau^{j} \partial_{j}(\phi \cdot \psi) & =\tau^{j}\left(\nabla_{a}\right)_{j} \phi \cdot \psi+\phi \cdot \mathscr{D} \psi \\
& =\tau^{j}\left(\nabla_{a}\right)_{j} \phi \cdot \psi+\phi \cdot g(\psi) .
\end{aligned}
$$

As just noted $g$ is a quadratic commutator, so the longitudinal components commute out and so by the first statement of the claim $g(\psi)$ is exponentially decaying. We can now apply, successively, cases A and B of Lemma C.1 to deduce the second statement of the claim.

Next we need to study the behaviour of the derivatives $\frac{\partial \psi}{\partial q}$, where $q$ is a local co-ordinate. Since the map $F$ is a local diffeomorphism, we know that $\frac{\partial \psi}{\partial q} \in H_{\Psi_{0}}$. The next result says that these have the same decay properties as $\psi$ itself:

Claim B. The derivative $\frac{\partial \psi}{\partial q}$ of the solution of the deformation equation just obtained has the following decay properties:

$$
\begin{gathered}
\left|\left[\phi, \frac{\partial \psi}{\partial q}\right]\right| \leqq c(\varepsilon) e^{-(1-\varepsilon)|x|}, \\
\left|\phi \cdot \frac{\partial \psi}{\partial q}\right| \leqq c(1+|x|)^{-2}
\end{gathered}
$$

Proof. The proof is almost identical to the proof of the previous claim.

For the reader's convenience we now summarise the definition of the circle bundle $M_{k}$ over the moduli space $N_{k}$ (see [AH88], to which the reader may also refer for an alternative description). This will clarify how it is that the local solution space produced in the last section is actually not a co-ordinate patch on $N_{k}$ but rather on the circle bundle $M_{k}$. The reason for this is of course the occurrence of the extra zero mode $n_{0}=\tau^{j}\left(\nabla_{a}\right)_{j} \phi$ corresponding to the action of the Higgs field as an infinitesimal gauge transformation. Thus we want to define a circle bundle whose fibre direction corresponds to the gauge action of the Higgs field. This is done via Hitchin's asymptotic isomorphism (see Sect. 6 of [Hit82]). The basic idea is as follow - corresponding to an $\mathrm{SU}(2)$ connection there is a $\mathbf{C}^{2}$ bundle. Let us take as basis for this the eigenvectors of $\Phi$ on a large sphere $S_{R}^{2}$ so that $\Phi$ has the form:

$$
\Phi \approx\left(\begin{array}{cc}
i & 0 \\
0 & -i
\end{array}\right) .
$$

Then in an appropriate gauge it turns out that the connection is asymptotically of the form 


$$
A \approx\left(\begin{array}{cc}
a & 0 \\
0 & -a
\end{array}\right)
$$

where $a$ is a connection on the sphere. As $R \rightarrow \infty$ this gives an asymptotic isomorphism $\alpha$ of the monopole with a direct sum $H^{k} \oplus H^{-k}$, where $H$ is Hopf's line bundle on the sphere. This is unique up to the circle action which is the automorphism group of this bundle. We see from the expression for $\Phi$ above that the action of the gauge transformations $e^{t \Phi}$ corresponds to a circle action

$$
\left(\begin{array}{cc}
e^{i t} & 0 \\
0 & e^{-i t}
\end{array}\right)
$$

on this bundle. Now -1 extends to the element -1 acting on the $\mathbf{C}^{2}$ bundle over $\mathbf{R}^{3}$. This is the only element of the circle $S^{1}$ which so extends to an automorphism of the monopole, since the monopoles are irreducible. Thus if we define a rigidified monopole to be made up of the gauge equivalence class of the monopole together with an asymptotic isomorphism $\alpha$ fixed up to sign, then the space of rigidified monopoles $M_{k}$ is acted on freely by $S^{1} /\{ \pm 1\}$, and has as quotient the space of gauge equivalence classes of monopoles. Furthermore given a monopole, all the gauge equivalent rigidified monopoles are obtained by considering the action of the gauge transformations $e^{t \Phi}$. This explains the construction of the local co-ordinate patches on $M_{k}$ in the previous section-deformation in the direction $n_{0}=D \Phi$ corresponds to the fibre direction in $M_{k}$.

The last thing we need to prove about our local solutions is that we can always choose the gauge such that the velocity vectors of our monopole $\Psi_{0}(\tau)$ satisfy the gauge orthogonality condition in Eq. 1.4 relative to $\Psi_{0}(\tau)$. Consider a smooth one parameter curve of monopoles

$$
\tau \rightarrow(a(x ; \tau), \phi(x ; \tau))
$$

lying in one of our co-ordinate patches. It will be convenient to introduce a one parameter family of gauge transformations to ensure that the velocity vectors of the transformed monopoles satisfy the condition of gauge orthogonality at each $\tau$. In this section we prove that this is possible. Thus we are searching for a family of gauge transformations

$$
\tau \rightarrow g(x ; \tau)
$$

(where for each $\tau, g(x, \tau)$ is $S U(2)$ valued), with the property that

$$
(\alpha(x ; \tau), \varphi(x ; \tau))
$$

is orthogonal to the gauge flow at each time:

$$
d_{\alpha} \cdot \dot{\alpha}+[\varphi, \dot{\varphi}]=0
$$

This requirement leads to the following equation:

$$
-\Delta_{\alpha}\left(g \dot{g}^{-1}\right)-\left[\varphi,\left[\varphi, g \dot{g}^{-1}\right]\right]=-d_{\alpha} \cdot\left(g \dot{a} g^{-1}\right)-\left[\varphi, g \dot{\phi} g^{-1}\right]
$$

Theorem 4.1. Consider a twice differentiable curve

$$
\tau \rightarrow(a(x ; \tau), \phi(x ; \tau)) \in M_{k}
$$

defined for $\tau \in(0, T)$, lying in one of the co-ordinate patches just discussed. Then for $\tau \in(0, T), x \in R^{3}$ there is a twice differentiable function $g(\tau, x) \in S U(2)$ which 
solves equation 4.3, and such that $g \rightarrow 1$ as $|x| \rightarrow \infty$. Also, for fixed $\tau, g$ is smooth as a function of $x$.

Proof. We take as initial iterate $g_{0}=1$; thereafter given the $i^{\text {th }}$ iterate $g_{i}$ we generate the $(i+1)^{\text {th }}$ by solving the equation:

$$
-\Delta_{\alpha(i)}\left(g_{i}+1 \dot{g}_{i+1}^{-1}\right)-\left[\varphi(i),\left[\varphi(i), g_{i+1} \dot{g}_{i+1}^{-1}\right]\right]=-d_{\alpha(i)} \cdot\left(g_{i} \dot{a} g_{i}^{-1}\right)-\left[\varphi(i), g_{i} \dot{\phi} g_{i}^{-1}\right],
$$

where

$$
(\alpha(i), \varphi(i))=\rho\left(g_{i}\right)(a, \phi) .
$$

This is not convenient to treat as it stands because the background monopole depends on $i$. So we introduce

$$
B_{i}=g_{i}^{-1} g_{i+1} \dot{g}_{i+1}^{-1} g_{i}
$$

then this solves the gauge transformed equation:

$$
-\Delta_{a} B_{i}-\left[\phi,\left[\phi, B_{i}\right]\right]=-\nabla_{a} \cdot \dot{a}-[\phi, \dot{\phi}],
$$

and so by Theorem A.8 we see that $B_{i}$ is uniformly bounded in $H_{s, \Psi_{0}}$ for all $i$. By differentiating the equation with respect to time we obtain the same conclusion for $\dot{B}_{i}$. We then obtain a subsequence which is weakly convergent for all times, and is in fact strongly convergent on all bounded subsets by Rellich's compactness theorem. We then define an SU(2) valued function inductively by solving the ordinary differential equation

$$
g_{i+1} \dot{g}_{i+1}^{-1}=g_{i} B_{i} g_{i}^{-1}
$$

and this gives the required solution of the equation.

Corollary 4.2. Let $q, q^{\prime}$ be two points on a co-ordinate patch of $M_{k}$, then the zero modes corresponding to the co-ordinate $q_{\mu}$ satisfy:

$$
\left|n_{\mu}(q)-n_{\mu}\left(q^{\prime}\right)\right|_{L^{2}} \leqq c\left|q-q^{\prime}\right|
$$

Proof. First of all we know that from the construction of the co-ordinate patches that

$$
\left|\frac{\partial \Psi_{0}}{\partial q_{\mu}}(q)-\frac{\partial \Psi_{0}}{\partial q_{\mu}}\left(q^{\prime}\right)\right|_{L^{2}} \leqq c\left|q-q^{\prime}\right| .
$$

Now the zero modes are obtained from $\frac{\partial \Psi_{0}}{\partial q_{\mu}}$ by applying the gauge transformations from the previous theorem. Applying the estimates from Theorem A.8 we then obtain the result.

\section{The Asymptotic Expansions}

In this section we show how to set up the asymptotic dynamics of multi-monopoles. We will then show that our expansions do indeed provide an approximate solution of the full equations in the slow motion limit. The equations we will consider therefore will be the Yang-Mills-Higgs equations on Minkowski space:

$$
-D_{i}^{2} A_{0}+D_{i} \partial_{t} A_{i}=-\left[\Phi, D_{0} \Phi\right]
$$




$$
\begin{gathered}
D_{0}^{2} A_{i}-D_{j}^{2} A_{i}-D_{0} \partial_{i} A_{0}+D_{j} \partial_{i} A_{j}=-\left[\Phi, D_{i} \Phi\right], \\
D_{0}^{2} \Phi-D_{1}^{2} \Phi-D_{2}^{2} \Phi-D_{3}^{2} \Phi=0 .
\end{gathered}
$$

We search for solutions which represent slowly evolving multi-monopoles, whose parameters vary on the time scale $\tau=\varepsilon t$. We will use $\dot{f}, \ddot{f}$ for $\frac{d f}{d \tau}, \frac{d^{2} f}{d \tau^{2}}$. Thus we search for a solution of the form

$$
\begin{aligned}
A_{0} & =\varepsilon^{3} \tilde{a}_{0}, \\
A_{i} & =\alpha_{i}(x ; t)+\varepsilon^{2} \tilde{a}_{i}, \\
\Phi & =\varphi+\varepsilon^{2} \tilde{\phi} .
\end{aligned}
$$

We can write the latter two equations as

$$
\Psi(t, x)=\Psi_{0}(x ; t)+\varepsilon^{2} \psi(t, x)
$$

and we choose the gauge such that $\dot{\Psi}_{0}$ and $\psi$ satisfy the gauge orthogonality condition with respect to $\Psi_{0}$ :

$$
\begin{aligned}
& \left(\nabla_{\alpha}\right)_{i} \dot{\alpha}_{i}+[\varphi, \dot{\varphi}]=0, \\
& \left(\nabla_{\alpha}\right)_{i} \tilde{a}_{i}+[\varphi, \tilde{\varphi}]=0,
\end{aligned}
$$

as discussed in the introduction. This is what makes $A_{0}=O\left(\varepsilon^{3}\right)$. It is a consequence of the local existence theorem proved in the appendix that it is possible to choose the gauge such that these two conditions are satisfied if $\varepsilon$ is small enough. We now calculate the equations for the error terms. Let $\Delta_{a}=\sum\left(\nabla_{a}\right)_{i}^{2}$ be the Laplacian with respect to a connection $a$, then we obtain:

$$
\begin{aligned}
-\Delta_{\alpha} \tilde{a}_{0}+\left[\phi,\left[\tilde{a}_{0}, \phi\right]\right]-2\left[\dot{\alpha}_{i}, \tilde{a}_{i}\right]-2[\dot{\varphi}, \tilde{\phi}] & =\varepsilon j_{0}, \\
\frac{\partial^{2} \tilde{a}_{i}}{\partial t^{2}}-\Delta_{\alpha} \tilde{a}_{i}+\left[\phi,\left[\tilde{a}_{i}, \phi\right]\right]-2\left[F_{i j}, \tilde{a}_{j}\right]-2\left[\left(\nabla_{\alpha}\right)_{i} \phi, \tilde{\phi}\right] & =-\ddot{\alpha}_{i}+\varepsilon_{j i}, \\
\frac{\partial^{2} \tilde{\phi}}{\partial t^{2}}-\Delta_{\alpha} \tilde{\phi}-\left(\nabla_{\alpha}\right)_{i}\left[\tilde{a}_{i}, \phi\right]-\left[\tilde{a}_{i},\left(\nabla_{\alpha}\right)_{i} \phi\right] & =-\ddot{\phi}+\varepsilon j_{4},
\end{aligned}
$$

where the nonlinear error terms are given in Appendix B. To see the structure of these equations it is best to introduce the variable $\psi$ defined in Eq. 3.1, in terms of which Eq. 5.10 and 5.11 can be written as the single equation

$$
\frac{\partial \psi}{\partial t^{2}}+L \psi=k-\varepsilon \mathscr{D}_{0}\left(\tilde{a}_{0, t}\right)+\varepsilon^{2} j,
$$

where $k=\left(-\ddot{\alpha}_{i}\right) \tau^{i}+-\ddot{\varphi}$, and the operator $L \Psi_{0}$ is defined by

$$
\int_{\mathbf{R}^{3}}\left(\psi, L \Psi_{0}, \phi\right)=\overline{\operatorname{Hess}}_{\alpha, \varphi}(\psi, \psi)=\int_{\mathbf{R}^{3}}\left|\mathscr{D} \Psi_{0} \psi\right|^{2},
$$

where $\overline{\text { Hess }}$ is the modified Hessian at $\Psi_{0}=(\alpha, \varphi)$ of the functional $\mathscr{E}$, as defined in Sect. 2.

The final step in the asymptotic argument is to find the appropriate equations for the evolution of the monopole parameters. This is done by requiring that $q(t)$ evolve in time such that 


$$
(\psi, n)_{L^{2}}=0 \quad \forall \quad n \in T_{\Psi_{0}} M_{k}
$$

is satisfied, as explained in the introduction. This is not very illuminating - to obtain a set of equations for the monopole parameters which are self-contained up to $O(\varepsilon)$ we differentiate this condition twice and substitute for $\psi_{t t}$. This leads to

$$
\left(\ddot{\Psi}_{0}, n\right)=-\varepsilon\left(\mathscr{D}_{\Psi_{0}}\left(\partial_{t} \tilde{a}_{0}\right), n\right)-2 \varepsilon\left(\psi_{t}, \dot{n}\right)+\varepsilon^{2}(j, n)-\varepsilon^{2}(\psi, \ddot{n}),
$$

where $(\cdot, \cdot)$ refers to $L^{2}$ inner product. To highest order this is the geodesic equation as suggested by Manton. If we use a co-ordinate system $q_{\mu}$ on our local space of solutions we can write $\dot{\Psi}_{0}=\dot{q}_{\mu} n_{\mu}$ and so the equation becomes:

$$
\ddot{q}_{\mu}=-\left(g^{-1}\right)_{v \mu}\left(\left(n_{v}, \dot{n}_{\lambda}\right) \dot{q}_{\lambda}-\varepsilon\left(\mathscr{D}_{\Psi_{0}}\left(\partial_{t} \tilde{a}_{0}\right), n_{v}\right)-2 \varepsilon\left(\psi_{t}, \dot{n}_{v}\right)+\varepsilon^{2}\left(j, n_{v}\right)-\varepsilon^{2}\left(\psi, \ddot{n}_{v}\right)\right) .
$$

We will be comparing the solution of this to the geodesic equation

$$
\ddot{q}_{\mu}^{0}=-\left(g^{-1}\right)_{v \mu}\left(\left(n_{v}, \dot{n}_{\lambda}\right) \dot{q}_{\lambda}^{0}\right. \text {. }
$$

Define $Z$ by

$$
Z=\left(Z^{1}, Z^{2}\right)=\left(\frac{q-q^{(0)}}{\varepsilon}, \frac{\dot{q}-\dot{q}^{(0)}}{\varepsilon}\right),
$$

then $Z$ satisfies an equation of the form

$$
\frac{d Z}{d t}=\varepsilon F\left(q^{(0)}, Z, \psi, \mathscr{D}_{\Psi_{0}}\left(\tilde{a}_{0, t}\right)\right),
$$

where

$$
|F| \leqq c\left(|q|,|\dot{q}|,|Z|,|\psi|_{2, \Psi_{0}},\left|\psi_{t}\right| \Psi_{0},\left|\tilde{a}_{0, t}\right| \Psi_{0}\right) .
$$

Remark. To estimate the term $(\psi, \ddot{n})$ we need the crucial result on the decay of $\ddot{n}$ modulo $T_{\Psi_{0}} M_{k}$ given by Lemma 3.5 , together with the fact that $\psi$ satisfies the dynamic condition with respect to $\Psi_{0}$ by choice of $q(t)$.

\section{The Basic Identities}

In this section we give the basic identities which are needed to obtain estimates for $\psi$. They can be thought of as approximate energy conservation laws which arise because we have linearised about monopole configurations which are slowly varying in time. We will omit the subscript on $\mathscr{D}$ with the understanding that this always means $\mathscr{D} \Psi_{0}(t)$, with $\Psi_{0}(t)=\Psi_{0}(x ; q(t))=(\alpha, \varphi)$. The basic energy is

$$
Q_{1}(t) \equiv \frac{1}{2} \int_{\mathbf{R}^{3}}\left(\psi_{t}, \psi_{t}\right)+(\mathscr{D} \psi, \mathscr{D} \psi) d x .
$$

If we differentiate this with respect to time, substitute for $\psi_{t t}$ and then integrate again we obtain:

$$
\begin{aligned}
Q_{1}(t)-Q_{1}(0)= & {\left[\left(\psi,-\ddot{\Psi}_{0}\right)_{L^{2}}\right]_{0}^{t}-\varepsilon \int_{0}^{t}\left(\psi, \Psi_{0}^{(3)}\right)+\left(\psi_{t}, \mathscr{D}\left(\partial_{t} \tilde{a}_{0}\right)+\varepsilon j\right)_{L^{2}} } \\
& +\varepsilon \int_{0}^{t}\left(\mathscr{D} \psi,-\tau^{j}\left[\dot{\alpha}_{j}, \psi\right]+[\dot{\varphi}, \psi]\right)_{L^{2}}
\end{aligned}
$$


where $\Psi_{0}^{(3)}=\frac{d^{3} \Psi_{0}}{d t^{3}}$. For the second estimate we consider $\psi=\mathscr{D} \psi$ which satisfies the equation:

$$
\begin{aligned}
\psi_{t t}^{\prime}+\mathscr{D} \mathscr{D}^{*} \psi^{\prime}= & -\mathscr{D} \ddot{\Psi}_{0}-\varepsilon \tau^{j}\left[\dot{\alpha}_{j}, \psi_{t}\right]+\varepsilon\left[\dot{\varphi}, \psi_{t}\right]-\varepsilon^{2} \tau^{j}\left[\ddot{\alpha}_{j}, \psi\right] \\
& +\varepsilon^{2}[\ddot{\varphi}, \psi]-\varepsilon \mathscr{D} \mathscr{D}\left(\partial_{t} \tilde{a}_{0}\right)+\varepsilon^{2} \mathscr{D} j \\
= & R_{0}+\varepsilon R_{1} .
\end{aligned}
$$

From this equation we will obtain a higher order energy identity for the quantity:

$$
Q_{2}(t) \equiv \frac{1}{2} \int_{\mathbf{R}^{3}}\left((\mathscr{D} \psi)_{t},(\mathscr{D} \psi)_{t}\right)+\left(\mathscr{D}^{*} \psi^{\prime}, \mathscr{D}^{*} \psi^{\prime}\right) \text {. }
$$

Proceeding as before we find the following identity:

$$
\begin{aligned}
Q_{2}(t)-Q_{2}(0)= & {\left[\left(\psi^{\prime},-\mathscr{D} \ddot{\Psi}_{0}\right)_{L^{2}}\right]_{0}^{t}-\varepsilon \int_{0}^{t}\left(\psi^{\prime}, \frac{d}{d \tau}\left(R_{0}\right)-\left(\psi_{t}^{\prime}, R_{1}\right)\right)_{L^{2}} d t } \\
& -\int_{0}^{t}\left(\mathscr{D}^{*} \psi^{\prime}, \tau^{j}\left[\dot{\alpha}_{j}, \psi^{\prime}\right]+\left[\dot{\varphi}, \psi^{\prime}\right]\right)_{L^{2}} d t
\end{aligned}
$$

The final identity we need is the energy identity for $\psi^{\prime \prime}=\mathscr{D}^{*} \psi^{\prime}=\mathscr{D}^{*} \mathscr{D} \psi$, which satisfies the equation:

$$
\begin{aligned}
\psi_{t t}^{\prime \prime}+\mathscr{D}^{*} \mathscr{D} \psi^{\prime \prime}= & \mathscr{D}^{*}\left(R_{0}+\varepsilon R_{1}-\varepsilon \tau^{j}\left[\dot{\alpha}_{j}, \psi_{t}^{\prime}\right]+\varepsilon\left[\dot{\varphi}, \psi_{t}^{\prime}\right]\right. \\
& -\varepsilon^{2} \tau^{j}\left[\ddot{\alpha}_{j}, \psi^{\prime}\right]+\varepsilon^{2}\left[\ddot{\varphi}, \psi^{\prime}\right], \\
= & \mathscr{D}^{*}\left(R_{0}+\varepsilon R_{1}\right)-\varepsilon S,
\end{aligned}
$$

which leads to an identity for

$$
Q_{3}(t) \equiv \frac{1}{2} \int_{\mathbf{R}^{3}}\left(\psi_{t}^{\prime \prime}, \psi_{t}^{\prime \prime}\right)+\left(\mathscr{D} \psi^{\prime \prime}, \mathscr{D} \psi^{\prime \prime}\right) d x .
$$

The identity for $Q_{3}$ is:

$$
\begin{aligned}
Q_{3}(t)-Q_{3}(0)= & {\left[\left(\psi^{\prime \prime}, \mathscr{D}^{*} R\right)_{L^{2}}\right]_{0}^{t}-\varepsilon \int_{0}^{t}\left(\psi^{\prime \prime}, \frac{d}{d \tau}\left(\mathscr{D}^{*} R_{0}\right)\right)_{L^{2}}+\left(\psi_{t}^{\prime \prime}, S-R_{1}\right)_{L^{2}} d t } \\
& +\int_{0}^{t}\left(\mathscr{D} \psi^{\prime \prime},-\tau^{j}\left[\dot{\alpha}_{j}, \psi^{\prime \prime}\right]+\left[\dot{\varphi}, \psi^{\prime \prime}\right]\right)_{L^{2}} d t
\end{aligned}
$$

\section{Co-ercive Estimates for the Conserved Quantities}

In this section we relate the quantities $Q_{i}$ to the norms defined in Appendix A. First of all a direct calculation shows that if $\Psi_{0}$ satisfies the Bogomolny equations, then for smooth compactly supported $u$ we have

$$
\left(\mathscr{D}^{*} u, \mathscr{D}^{*} u\right)_{L^{2}}=|u|^{2} \Psi_{0} .
$$

Theorem 7.1. There is a positive number $\gamma=\gamma(k)$ such that the following is true. For any monopole $\Psi_{0}$ of charge $k$ and any smooth, compactly supported section $u$ which is $L^{2}$ - orthogonal to $T_{\Psi_{0}} M_{k}$ we have 


$$
\left|\mathscr{D} \Psi_{0} u\right|_{L^{2}} \geqq \gamma|u|_{\Psi_{0}} .
$$

Proof. The proof is an adaptation of the proof of a result of Taubes ([Tau83]) which is given as Lemma A.3 in our Appendix A. The crucial new input is Theorem A.10. The result follows from the following two lemmas:

Lemma 7.2. Let $u^{i}$ be a sequence of smooth, compactly supported sections which have the property that $\left(u^{i}, n\right)_{L^{2}}=0 \quad \forall n \in T_{\Psi_{0}} M_{k}$. Then for each monopole $\Psi_{0}$ there is a number $\gamma\left(\Psi_{0}\right)$ such that

$$
\left|\mathscr{D} \Psi_{0} u^{i}\right|_{L^{2}} \geqq \gamma\left(\Psi_{0}\right)\left|u^{i}\right|_{\Psi_{0}} .
$$

Proof. First of all recall from Lemma A.3 that $\mathscr{D}$ is a Fredholm operator from $H_{\Phi_{0}} \rightarrow L^{2}$. Now consider a minimising sequence with the properties

(i) $\left|\mathscr{D} u^{i}\right|_{L^{2}} \rightarrow 0$,

(ii) $\left|u^{i}\right|_{\Psi_{0}}=1$

(iii) $\left(u^{i}, n\right)_{L^{2}}=0 \forall n \in T_{\Psi_{0}} M_{k}$.

We first of all show that there is a number $\gamma\left(\Psi_{0}\right)$ with the required property by using this sequence to produce a contradiction to Lemma A.3. We then show that the dependence of $\gamma$ on the background configuration can be removed. To obtain the contradiction we introduce a new sequence

$$
v^{i}=u^{i}+n^{i} \text { where }\left(v^{i}, n\right)_{\Psi_{0}}=0 \quad \forall n \in T_{\Psi_{0}} M_{k}
$$

so that $n^{i}=\sum\left(v^{i}, n_{\mu}\right) n_{\mu}$. Notice that on account of the third condition on the $u^{i}$ that $v^{i} \neq 0$. There are two possibilities - if $\left|v^{i}\right|_{\Psi_{0}} \geqq \varepsilon>0$ as $i \rightarrow \infty$, then we can renormalise to produce a sequence such that:

(i) $\left|\mathscr{D} \tilde{v}^{i}\right|_{L^{2}} \rightarrow 0$,

(ii) $\left|\tilde{v}^{i}\right|_{\Psi_{0}}=1$,

(iii) $\left(\tilde{v}^{i}, n\right)_{L^{2}}=0 \forall n \in T_{\Psi_{0}} M_{k}$.

This contradicts Lemma A.3 and is therefore impossible. The second possibility is that $\left|v^{i}\right|_{\Psi_{0}} \rightarrow 0$ as $i \rightarrow \infty$. This is in fact impossible since we know from Theorem A.10 that we can write $n_{\mu}=\mathscr{D} f_{\mu}, f_{\mu} \in L^{2}$, so that in this case

$$
n^{i}=\sum\left(v^{i}, n_{\mu}\right) n_{\mu}=\sum\left(\mathscr{D}^{*} v^{i}, f_{\mu}\right) n_{\mu}
$$

goes to zero in $H_{\Psi_{0}}$ by Holder's inequality since $\left|\mathscr{D}^{*} v\right|_{L^{2}} \leqq c|v| \Psi_{0}$. Taken together this imples that $\left|u^{i}\right|_{\Psi_{0}} \rightarrow 0$ which contradicts the second assumption. Therefore both possibilities have been ruled out and so the lemma is proved.

Lemma 7.3. There exists a number $\gamma$ such that $\gamma\left(\Psi_{0}\right) \geqq \gamma$ for all monopoles of given charge $k$.

Proof. We prove this by first showing that $\gamma\left(\Psi_{0}\right)$ is a continuous function on the moduli space, and then showing that outside large bounded sets it is bounded below. The existence of such a bound comes from the fact that as two monopoles separate they look more and more like a superposition of two single monopoles. But for the case of a single monopole $\gamma\left(\Psi_{0}\right)=\gamma$ is fixed by translation invariance. Therefore we expect that for two monopoles the value of $\gamma$ will approach that of a single monopole as the separation increases without limit. We will now be precise: 
Claim A.

$$
\gamma\left(\Psi_{0}\right)=\inf \left\{\left|\mathscr{D}_{\Psi_{0}} \psi\right|_{L^{2}}:|\psi|_{\Psi_{0}}=1(\psi, n)_{L^{2}}=0 \quad \forall n \in T_{\Psi_{0}} M_{k}\right\}
$$

is continuous.

Proof of Claim. This follows from two facts. First of all we know from Corollary 4.2 that the process of projecting $L^{2}$-orthogonal to $T_{\Psi_{0}(q)} M_{k}$ is continuous with respect to $q$. Also for fixed $\psi \in H_{\Psi_{0}}$ the functional $\left|\mathscr{D} \Psi_{0} \psi\right|_{L^{2}}$ is a continuous function of $q$. The result is now immediate.

Claim B. Let $q_{i} \rightarrow \infty$ be any sequence of monopoles tending to infinity on $M_{2}$, then there exists a universal $\gamma>0$ such that

$$
\gamma\left(\Psi_{0}\left(q_{i}\right)\right) \geqq \gamma>0 .
$$

Proof. We will derive a contradiction to the possibility that $\gamma\left(\Psi_{0}\left(q_{i}\right)\right) \rightarrow 0$. If this were so we would have a sequence of sections $u^{i}, L^{2}$-orthogonal to the zero modes of $\Psi_{0}\left(q_{i}\right)$, with $\left|u^{i}\right|_{\Psi_{0}\left(q_{i}\right)}=1$ and $\left|\mathscr{D}_{\Psi_{0}\left(q_{i}\right)} u^{i}\right|_{L^{2}} \rightarrow 0$. Now it is known from Theorem A.11 that there are sequences of points $x_{i}^{\alpha}$ and gauge transformations $g_{i}^{\alpha} \in C^{\infty}\left(\mathbf{R}^{3} ; \mathrm{SU}(2)\right)$ such that $P^{i}=\rho\left(g_{i}^{\alpha}\right)\left(\Psi_{0}^{i}\left(\cdot-x_{i}^{\alpha}\right)\right.$ converges, subsequentially, to a $k_{i}$-monopole $Q$ in the following sense - on any compact subset of $\mathbf{R}^{3}$ all derivatives converge uniformly. We will refer to the well-separated $k_{i}$-monopoles as the emerging monopoles for the sequence, since as the sequence goes outside larger and larger compact sets on $M_{k}$ the solution looks locally more and more like a superposition of well-separated monopoles. This picture of the asymptotic behaviour of the moduli space is discussed in [AH88, page 24] and [Tau85]. Applying these same translations and gauge transformations to $u^{i}$ we obtain a sequence $w^{i}, L^{2}$-orthogonal to all the zero modes of $P^{i}$, such that $\left|w^{i}\right|_{P^{i}}=1$ and

$$
\left|\mathscr{D}_{P^{i}} w^{i}\right|_{L^{2}} \rightarrow 0
$$

Now using the uniform convergence $P^{i} \rightarrow Q$ on compact sets we can deduce that $w^{i}$ is uniformly bounded in $H_{Q}(K)$ for each compact set $K$, where this is the Sobolev space obtained by completing the smooth sections $\left.C^{\infty}\left(\mathbf{R}^{3}\right) ; s u(2) \otimes \mathbf{H}\right)$ with respect to the norm obtained by restricting $|\cdot|_{Q}$ to $K$. By a diagonal argument we obtain a subsequence which converges weakly to a limit $w$ in $H_{Q}(K)$ for each compact set $K$. We next need to show that we may assume that $w \neq 0-$ this is because as discussed above outside sufficently large compact subsets the moduli space consists of collections of monopoles at large separations from one another. Furthermore, in between these emergent monopoles, as we called them above, it is known from Taubes' cluster theorems (see [Tau85, Section C]) that $F$ and $D \Phi$ decrease to zero with a Coulomb tail. Therefore if all the minimising sequence $w^{i}$ were concentrating away from the emerging monopoles as $i \rightarrow \infty$ we would contradict 7.1 on account of Eq. A.22. Therefore the sequence must be accumulating in the neighbourhood of one of the emergent monopoles and without loss of generality this may be taken to be $Q$. We next claim that the limit satisfies $\mathscr{D} \Psi_{0} w=0$. Since this is an elliptic equation we only need show that $w$ is a solution in the weak sense, i.e. for all compactly supported smooth test functions $s$

$$
\left(\mathscr{D}_{Q}^{*} s, w\right)_{L^{2}}=0
$$


But by Rellich $w^{i} \rightarrow w$ strongly on compact sets, so this follows from $\mathscr{D}_{p i} w^{i}=$ 0 . But also we can deduce from the strong convergence on compact subsets that $|w|_{H_{Q}(K)} \leqq 1$ for all compact sets $K$ and therefore $w \in H_{q}\left(\mathbf{R}^{3}\right)$ so $w=n \in T_{Q} M_{k}$. This then gives a contradiction to the fact that $w^{i}$ were orthogonal to all the zero modes of $P^{i}$ via Corollary A.12.

From Theorem 7.1 follows the fact that $Q_{1}+Q_{2}+Q_{3}$ is a good norm for the solution. We now state this using the spaces $H_{r, \Psi_{0}}$ defined in the Appendix A.

Theorem 7.4. Let $t \rightarrow \Psi_{0}(t)=(a(x ; t), \phi(x ; t))$ be a curve in one of the local solution spaces. Then there is a number $k$, depending on $\left|\dot{\Psi}_{0}\right| \Psi_{0}$ and $\left|F_{a}\right| \Psi_{0}$, such that for $\left(\psi, \psi_{t}\right) \in\left(H_{3, \Psi_{0}} \cap L^{2}\right) \oplus\left(H_{2, \Psi_{0}} \cap L^{2}\right)$ with $\psi$ being $L^{2}$ orthogonal to $T_{\Psi_{0}} M_{k}$, we have:

$$
Q_{1}+Q_{2}+Q_{3} \geqq \kappa\left(|\psi|_{3, \Psi_{0}(t)}^{2}+\left|\psi_{t}\right|_{2, \Psi_{0}(t)}^{2}+\left|\psi_{t}\right|_{L^{2}}^{2}\right)
$$

Proof. From the previous lemma we know that

$$
|\psi|_{\Psi_{0}(t)}^{2}+|\psi|_{L^{2}}^{2} \leqq 2 \kappa_{1} Q_{1}
$$

where $\kappa_{1}=\max \left(1, \gamma^{-2}\right)$ and $\gamma$ is as in Theorem 7.1. Now $(\mathscr{D} \psi)_{t}=\mathscr{D} \psi_{t}$ $\tau^{j}\left[\dot{a}_{j}, \psi\right]+[\dot{\phi}, \psi]$ so we get

$$
\left|\mathscr{D} \psi_{t}\right|_{L^{2}}^{2} \leqq c\left(Q_{1}+Q_{2}\right)
$$

by an application of Lemma A.4. But since $\left|\psi_{t}\right|_{L^{2}}^{2} \leqq 2 Q_{1}$ we obtain

$$
\left|\psi_{t}\right|_{L^{2}}^{2}+\left|\psi_{t}\right|_{1, \Psi_{0}(t)}^{2} \leqq c\left(Q_{1}+Q_{2}\right) .
$$

Next we notice that by the previous lemma

$$
\sum_{i=1}^{3}\left|\left(\nabla_{a}\right)_{i} \mathscr{D} \psi\right|_{L^{2}}^{2} \leqq c Q_{2},
$$

so that using the identity

$$
\left(\nabla_{a}\right)_{i}(\mathscr{D} \psi)-\mathscr{D}\left(\left(\nabla_{a}\right)_{i} \psi\right)=-\tau^{j}\left[F_{i j}, \psi\right]+\left[\left(\nabla_{a}\right)_{i} \phi, \psi\right],
$$

we see that

$$
|\psi|_{2, \Psi_{0}(t)}^{2} \leqq c\left(Q_{2}+Q_{1}\right) \text {. }
$$

To complete the proof we apply these commutator arguments to $\psi^{\prime}$ and $\psi^{\prime \prime}$, noting that $\psi^{\prime \prime}=\mathscr{D}^{*} \psi^{\prime}$ is automatically $L^{2}$-orthogonal to $T_{\Psi_{0}} M_{k}$ and so the previous lemma can be applied to obtain information from $Q_{3}$.

\section{Proof of Main Theorem}

We are now in a position to prove that the asymptotic expansions do indeed provide a good approximation to the full equations for small $\varepsilon$. The idea is that we can produce local solutions with a continuation theorem with respect to a norm which 
is equivalent to $Q_{1}^{1 / 2}+Q_{2}^{1 / 2}+Q_{3}^{1 / 2}$. But the identities of Sect. 6 then allow this solution to be continued for times of $O\left(\frac{1}{\varepsilon}\right)$. Let us introduce the following norms:

$$
\begin{gathered}
\|\psi(T)\|_{1}=\max _{0 \leqq t \leqq T}\left(|\psi|_{3, \Psi_{0}(0)}+\left|\psi_{t}\right|_{2, \Psi_{0}(0)}\right), \\
\left\|\tilde{a}_{0}(T)\right\|_{2}=\max _{0 \leqq t \leqq T}\left(\left|\tilde{a}_{0}\right|_{4, \Psi_{0}(0)}+\left|\tilde{a}_{0, t}\right|_{3, \Psi_{0}(0)}\right), \\
\|Z(T)\|_{3}=\max _{0 \leqq t \leqq T}|\dot{q}(t)|,
\end{gathered}
$$

where $\Psi_{0}(0)=(a(0), \phi(0))$ is the initial monopole. As above we will write $\Psi_{0}(t)=$ $\Psi_{0}(x ; q(t))=(\alpha, \varphi)$.

Theorem 8.1. Consider the system of equations

$$
\begin{gathered}
\psi_{t t}+L_{\Psi_{0}(t)} \psi=-\ddot{\Psi}_{0}-\varepsilon \mathscr{D} \Psi_{0}(t) \\
\left.-\Delta_{\alpha} \partial_{t} \tilde{a}_{0}\right)+\varepsilon^{2} j^{\prime} \\
\left.\left.\frac{d Z}{d t}=\varepsilon F\left(\tilde{a}_{0}, \varphi\right]\right]=2\left[\dot{\alpha}_{k}, \tilde{a}_{k}\right]+\varepsilon, \psi, \mathscr{D}\left(\partial_{t} \tilde{a}_{0}\right)\right)
\end{gathered}
$$

derived in Sect. 5, with initial data $\psi(0), \psi_{t}(0), Z(0)$ satisfying:

(i) $\psi(0) \in L^{2}$,

(ii) $(\psi(0), n)_{L^{2}}=0 \quad \forall n \in T_{\Psi_{0}(0)} M_{k}$,

(iii) $\|\psi(0)\|_{1}+\|Z(0)\|_{3} \leqq \frac{\Gamma}{2}$,

(iv) $\nabla_{a(0)} \cdot \tilde{a}(0)+[\psi(0), \tilde{\psi}(0)]=0$.

Then there exist numbers $\varepsilon_{*}, T_{\text {loc }}$, depending on $\Gamma$, such that for $\varepsilon<\varepsilon_{*}, t<T_{\text {loc }}$ there exists a unique solution which satisfies the following bounds:

(a) $\left\|\psi\left(T_{\text {loc }}\right)\right\|_{1}+\left\|Z\left(T_{\text {loc }}\right)\right\|_{3} \leqq \Gamma$,

(b) there exist numbers $A(\Gamma), B(\Gamma)$ such that

$$
\left\|\tilde{a}_{0}\right\|_{2} \leqq A(\Gamma) \quad\left|\psi_{t t}(t)\right|_{1, \Psi_{0}(0)} \leqq B(\Gamma) .
$$

The solution has the following regularity: the maps

$$
\begin{aligned}
t & \mapsto\left(\psi, \psi_{t}\right) \in H_{1, \Psi_{0}(0)} \oplus L^{2} \\
t & \mapsto \tilde{a}_{0} \in H_{2, \Psi_{0}(0)} \\
t & \mapsto(Z, \dot{Z}) \in C^{1}\left(0, T_{\text {loc }}\right) \oplus C\left(0, T_{\text {loc }}\right)
\end{aligned}
$$

are strongly differentiable, while the maps

$$
\begin{aligned}
& t \mapsto\left(\psi, \psi_{t}\right) \in H_{3, \Psi_{0}(0)} \oplus H_{2, \Psi_{0}(0)}, \\
& t \mapsto\left(\tilde{a}_{0}, \tilde{a}_{0, t}\right) \in H_{4, \Psi_{0}(0)} \oplus H_{3, \Psi_{0}(0)}
\end{aligned}
$$

are continuous. The solution satisfies the identities given in Sect. 6 and also the orthogonality conditions

(i) $(\psi, n)_{L^{2}}=0 \quad \forall n \in T_{\Psi_{0}(t)} M_{k}$,

(ii) $\nabla_{\alpha(t)} \cdot \tilde{a}(t)+[\varphi(t), \tilde{\phi}(t)]=0$ a.e.

Proof. This is given in Appendix E. 
Now to prove the theorem we use the identities of Sect. 6 to obtain an a priori estimate which allows us to continue these local solutions for times of $O\left(\frac{1}{\varepsilon}\right)$ with bounds independent of $\varepsilon$.

Lemma 8.2. Consider a local solution given by the previous result. Then the higher order energy defined by

$$
Q(T) \equiv \max _{0 \leqq t \leqq T}\left(Q_{1}+Q_{2}+Q_{3}\right)
$$

satisfies

$$
Q(T) \leqq Q(0)+c Q^{1 / 2}(T)(1+\varepsilon c(\Gamma))+\varepsilon T c(\Gamma) .
$$

Proof. We start off with some observations on the size of $q$ and its derivatives for these solutions. From Eq. 5 we see that using Lemma 3.5 and the result given in Appendix B that

$$
|\ddot{q}| \leqq c+\varepsilon c(\Gamma)
$$

while if we differentiate this equation we see that

$$
\left|\frac{d^{3} q}{d \tau^{3}}\right| \leqq c(\Gamma) .
$$

Given this we can estimate the terms. Most of these are done in the obvious fashion - the crucial point is that the nonlinear error terms are estimable using only the $\psi_{0}$ norm (see Appendix B) and the linear terms are estimated as

$$
\left|\int\left(\psi, \ddot{\Psi}_{0}\right) d x\right| \leqq\left.|\psi|_{L^{6}}|| \Pi\left(\ddot{\Psi}_{0}\right)\right|_{L^{6 / 5}}
$$

where we use the fact that since $\dot{\Psi}_{0} \in T_{\Psi_{0}} M_{k}$ we can apply Lemma 3.5 to obtain $\Pi\left(\ddot{\Psi}_{0}\right)$, the component of $\ddot{\Psi}_{0}$ which decays rapidly according to Lemma 3.5. Here we have used the fact that

$$
(\psi, n)_{L^{2}}=0 \quad \forall n \in T_{\Psi_{0}} M_{k},
$$

so the other component of $\ddot{\Psi}_{0}$ drops out. The other terms are treated using Lemma B.1 from the second appendix, together with the observations above on $q$.

We can now prove the main theorem. We will search for solutions satisfying

$$
Q^{1 / 2}(t) \leqq L
$$

for suitable $L$, with the initial data satisfying $Q^{1 / 2}(0) \leqq l<L$. By the local existence theorem for a short time condition 8.1 will certainly hold by continuity. By Lemma 7.4 there exists a $\kappa=\kappa(L)$ such that $\|\psi(t)\|_{1} \leqq \kappa^{-1} L$. So we get a corresponding solution from the local existence theorem which will satisfy

$$
Q(t) \leqq Q(0)+c Q^{1 / 2}(t)\left(1+\varepsilon c\left(\kappa^{-1} L\right)\right)+\varepsilon t c\left(\kappa^{-1} L\right) .
$$

From this we see that in fact $Q^{1 / 2} \leqq L$ for a time of order $\frac{1}{\varepsilon}$ and so the solution can be continued throughout such a time interval by successive application of the local existence theorem. To complete the proof of the theorem we observe that by direct calculation our solution will satisfy the gauge conditions which were required 
in Sect. 5 and therefore our solution will be a solution to the Yang-Mills-Higgs equations of the required form.

Remark. The solution is expected to be unique up to gauge invariance.

\section{A. Appendix A: Some Basic Facts}

In this appendix we collect together various definitions and results, mostly due to Taubes, needed in the paper. Unless otherwise stated all norm and integrals are for $\mathbf{R}^{3}$. First of all we introduce the function spaces used in this paper:

Definition. Let $\Psi_{0}=(a, \phi)$ be a monopole configuration. The Hilbert space $H_{\Psi_{0}}$ is the completion of the smooth, compactly supported sections $\Gamma(s u(2) \otimes \mathbf{H})$ with respect to the norm

$$
|\psi|_{\Psi_{0}}^{2} \equiv\left|\nabla_{a} \psi\right|_{L^{2}}^{2}+|[\phi, \psi]|_{L^{2}}^{2}
$$

The Hilbert space $H_{r, \Psi_{0}}$ is the completion of the smooth, compactly supported sections with respect to the norm

$$
|\psi|_{r, \Psi_{0}}^{2} \equiv \sum_{|\alpha|=1}^{r}\left|\nabla_{a}^{\alpha} \psi\right|_{L^{2}}^{2}+|[\phi, \psi]|_{L^{2}}^{2},
$$

where $\alpha$ is a multi-index. Finally we will use the following notation for the induced norm on bounded linear functionals of $H_{\Psi_{0}}$ :

$$
|f|_{*, \Psi_{0}}=\max _{u \in H_{\Psi_{0}}} \frac{|(f, u)|}{|u| \Psi_{0}} .
$$

Kato's lemma (see [JT82, Sect. 6.6]) gives a connection between the ordinary Sobolev spaces and those defined with covariant derivatives:

Lemma A.1 Let $\Psi$ be a section of a smooth section of a vector bundle on Euclidean space with smooth connection a. Then

$$
\int|\nabla| \Psi \|^{2} d x \leqq \int\left(D_{a} \Psi, D_{a} \Psi\right) d x
$$

Proof. See [JT82].

This leads us to the following gauge invariant Sobolev inequalities

Theorem A.2.

$$
\begin{aligned}
|\psi|_{L^{6}} & \leqq c|\psi|_{\Psi_{0}}, \\
|\psi|_{L^{\infty}} & \leqq c|\psi|_{2, \Psi_{0}} .
\end{aligned}
$$

Theorem A.3. The operator $\mathscr{D}_{\Psi_{0}} \equiv-\tau^{j} \nabla_{a}+[\phi, \cdot]$ is a Fredholm operator from $H_{\Psi_{0}}$ to $L^{2}$ with $4 k$ dimensional kernel $T_{\Psi_{0}} M_{k}$ when $\Psi_{0}$ represents a k-monopole. There is a number $\gamma\left(\Psi_{0}\right)$ such that

$$
|\psi|_{\Psi_{0}} \leqq \gamma^{-1}\left|\mathscr{D}_{\Psi_{0}} \psi\right|_{L^{2}}
$$

for any $\psi \in H_{\Psi_{0}}$ such that $(\psi, n)_{\Psi_{0}}=0$ for all $n \in T_{\Psi_{0}} M_{k}$. 
Proof. See Lemma 7.6 in [Tau83].

Lemma A.4. Let $\Psi_{0}=(a, \phi)$ be a configuration in which $|\phi| \rightarrow 1$ uniformly as $|x| \rightarrow \infty$. Then there exists a number $M\left(\Psi_{0}\right)$ such that for any $\psi_{1}, \psi_{2} \in H_{\Psi_{0}}$, and any $\delta, \varepsilon \in 1,2,3,4$,

$$
\begin{gathered}
\left|\left[\psi_{1}^{\delta}, \psi_{2}^{\varepsilon}\right]\right|_{L^{2}} \leqq M\left(\Psi_{0}\right)\left|\psi_{1}\right| \Psi_{0}\left|\psi_{2}\right| \Psi_{0} \\
\mid\left[\psi_{1}^{\delta},\left.\left[\psi_{2}^{\varepsilon}, \phi\right]\right|_{L^{2}} \leqq\left|\psi_{1}\right| \Psi_{0}\left|\psi_{2}\right| \Psi_{0} .\right.
\end{gathered}
$$

The number $M\left(\Psi_{0}\right)$ depends only on the measure of the set on which $|\phi(x)|<\frac{1}{2}$. Proof. See Lemma 6.6 in [Tau82].

Lemma A.5. Let $u \in L^{p}\left(\mathbf{R}^{d}\right)$ and $\nabla u \in L^{p}\left(\mathbf{R}^{d}\right)$ with $p>d$. Then $u$ decays uniformly to zero:

$$
\lim _{R \rightarrow \infty} \sup _{|x|=R}|u(x)|=0 \text {. }
$$

Proof. See Lemma 7.5 in Chapter three of [JT82].

Lemma A.6. Let $u$ and $v$ be smooth functions, both decaying uniformly to zero as $|x| \rightarrow \infty$, and that $u$ is positive. Assume further that $q$ is a smooth function which is exponentially decaying

$$
|q(x)| \leqq M_{1} e^{-\sigma|x|}
$$

Finally assume that

$$
-\Delta u+\left(1+\frac{\varepsilon}{2}\right)(1-v) u \leqq|q| .
$$

Then for any $\delta>0$ there is a number $M(\delta)$ such that for sufficently large $|x|$,

$$
|u(x)| \leqq M(\delta) e^{-(1-\delta) m|x|},
$$

where $m=\min \left\{1+\frac{\varepsilon}{2}, \sigma\right\}$.

Proof. The proof is as in chapter three of [JT82]. We consider a comparison function

$$
s=c M_{1} e^{-\bar{m}|x|} \text {. }
$$

Then choosing $\bar{m}<\sigma$ we can ensure that for sufficiently large $|x|$,

$$
\Delta s \leqq \bar{m}^{2} s-c|q| \text {. }
$$

Thus we obtain for $w=u-s$ :

$$
-\Delta w+\left(1+\frac{\varepsilon}{2}\right)(1-v) w \leqq\left(\bar{m}^{2}-\left(1+\frac{\varepsilon}{2}\right)(1-v)\right) s-c|q|+|q|
$$

Now for sufficently large $|x|$ we can ensure that $|v|<\frac{\varepsilon}{2}$, and thus that if $c>1$ and $\bar{m}<\left(1+\frac{\varepsilon}{2}\right)$ then 
Geodesic Approximation for Yang-Mills-Higgs Equations

$$
-\Delta w+\left(1+\frac{\varepsilon}{2}\right)(1-v) w \leqq 0 .
$$

Thus to complete the proof we just have to apply the maximum principle to a region $|x|>R(\delta)$ and choose $c$ large enough that $|u|<s$ on $|x|=R(\delta)$.

Theorem A.7. Let a be a smooth connection on a vector bundle over $\mathbf{R}^{3}$ and assume the associated curvature is square integrable. Let $u$ be a smooth square integrable section of $E$ which satisfies an equation of the form

$$
-\nabla_{a}^{2} u=\Lambda u+v
$$

where $\Lambda$ is a matrix with square integrable entries and $v$ is square integrable. Then $|u| \in L^{p, 2}\left(\mathbf{R}^{3}\right)$ for $2 \leqq p \leqq 6$; in particular $|u|$ and its first and second derivatives are all square integrable.

Proof. See Chapter five, Sect. seven in [JT82].

We next discuss some elliptic equations which turn up frequently in the paper. First of all we will discuss the equation

$$
-\Delta_{a} u+[\phi,[\phi, u]]=f
$$

for a fixed background $\Psi_{0}=(a, \phi)$.

Theorem A.8. Assume that $f$ is an su(2) valued function such that

$$
\begin{aligned}
& \text { (a) }\left|\int(f, u)\right| \leqq|f|^{*}|u| \Psi_{0}, \\
& \text { (b) }|f| \in L^{2}, \\
& \text { (c) } f \in H_{s, \Psi_{0}} \text { for some } s \geqq 0,
\end{aligned}
$$

then there exists a unique continuous, solution to A.14 which tends to zero as $|x| \rightarrow \infty$. It satisfies estimates

$$
|u|_{2+s, \Psi_{0}} \leqq c\left(|f|_{s, \Psi_{0}},|f|^{*},|f|_{L^{2}},|F|_{s+1, \Psi_{0}},|F|_{L^{2}}\right)
$$

where $F$ is the curvature of $a$.

Proof.

Step One. The functional $\int\left|\nabla_{a} u\right|^{2}+|[\phi, u]|^{2}$ is a norm on $H_{\Psi_{0}}$, so since $f$ defines a bounded linear functional on this space we get by the Riesz representation theorem an element $u \in H_{\Psi_{0}}$ such that for every $v \in H_{\Psi_{0}}$,

$$
\int\left(\nabla_{a} u, \nabla_{a} v\right)+\int([\phi, u],[\phi, v])=\int(f, v),
$$

so $u$ will be weak solution.

Step Two. We next differentiate the equation to obtain an estimate in $H_{2, \Psi_{0}}$. So let $u_{i}=\left(\nabla_{a}\right)_{i} u$, then it satisfies the equation

$-\Delta_{a} u_{i}+\left[\phi,\left[\phi, u_{i}\right]\right]=\left(\nabla_{a}\right)_{i} f-\left(\nabla_{a}\right)_{i}\left[F_{i j}, u\right]-\left[\left(\nabla_{a}\right)_{i} \phi,[\phi, u]\right]-\left[\phi,\left[\left(\nabla_{a}\right)_{i} \phi, u\right]\right]$. 
Now multiply this equation by $u_{i}$ and integrate by parts; the right-hand side is estimated by:

$$
\begin{aligned}
& \mid \int\left(u_{i},\left.\left(\nabla_{a}\right)_{i} f|\leqq| f\right|_{L_{2}}\left|u_{i}\right| \Psi_{0},\right. \\
& \left|\int\left(\left(\nabla_{a}\right)_{i}\left[F_{i j}, u\right], u_{i}\right)\right| \leqq c|F| \Psi_{0}|u| \Psi_{0}\left|u_{i}\right| \Psi_{0}, \\
& \mid \int\left(u_{i},\left.\left[\left(\nabla_{a}\right)_{i \phi},[\phi, u]\right)|\leqq| D_{a \phi}\left|\Psi_{0}\right| u\right|_{\Psi_{0}}\left|u_{i}\right| \Psi_{0},\right.
\end{aligned}
$$

and similarly for the fourth term. This leads to an estimate of the form

$$
|u|_{2, \Psi_{0}} \leqq c\left(|f|^{*},|f|_{L^{2}},|F|_{\Psi_{0}}\right) .
$$

Step Three. We continue the process of the last section indefinitely to obtain the stated estimate. We then know by A.5 that the solution decays uniformly to zero, and the theorem is proved.

Remark. It is shown how to justify fully the integration by parts calculations in these proofs in Sect. 5.7 of [JT82].

Example. Using Lemma A.4 we can apply this in the following two cases:

(i) If $f=[g, h]$ with $g \in H_{\Psi_{0}}, h \in L^{2}$.

(ii) If $f=\left[f_{1},\left[f_{2}, f_{3}\right]\right]$ with all $f_{i} \in H_{\Psi_{0}}$.

We also need to apply this to solving the equation

$$
\mathscr{D}_{\Psi_{0}}^{*} \mathscr{D}_{\Psi_{0}} u=f
$$

Here the associated quadratic form is $|\mathscr{D} u|_{L^{2}}^{2}$ which is only equivalent to the $H_{\Psi_{0}}$ norm on the subspace orthogonal to $T_{\Psi_{0}} M_{k}$. Apart from this everything works exactly as in the previous theorem, as long as $f$ satisfies the Fredholm conditions $\left(f, n_{\mu}\right)_{L^{2}}=0$, on account of the following Lichnerowicz formula:

$$
\mathscr{D}^{*} \mathscr{D}_{u}=-\Delta_{a} u-[\phi,[\phi, u]]+\frac{1}{2} \tau^{i} \varepsilon_{i j k}\left[F_{j k}, u\right]-\tau^{i}\left[\left(\nabla_{a}\right)_{i \phi}, u\right] .
$$

This leads to the identical theorem in this case:

Theorem A.9. Assume that $f$ satisfies the conditions in the previous theorem, and is $L^{2}$-orthogonal to $T_{\Psi_{0}} M_{k}$, then there exists a unique, continuous solution to A.21 which is $H_{\Psi_{0}}$-orthogonal to $T_{\Psi_{0}} M_{k}$ and tends to zero as $|x| \rightarrow \infty$. It satisfies estimates

$$
|u|_{2+s, \Psi_{0}} \leqq c\left(|f|_{s, \Psi_{0}},|f|^{*},|f|_{L^{2}},|F|_{s+1, \Psi_{0}},|F|_{L^{2}}\right)
$$

where $F$ is the curvature of $a$.

Theorem A.10. Let $n \in T_{\Psi_{0}} M_{k}$, then there exists $f \in L^{2} \cap H_{\Psi_{0}}$ such that

$$
\mathscr{D} \Psi_{0} f=n \text {. }
$$

Proof. The existence of such an $f \in H_{\Psi_{0}}$ is immediate from the results just given. We have to show that $f \in L^{2}$. We know that $[\phi, f] \in L^{2}$, so we have to investigate $\phi \cdot f$. The crucial point is the equation:

$$
\left.\tau^{j} \partial_{j}(\phi \cdot f)=\tau^{j}\left(\left(\nabla_{a}\right)_{j} \phi\right) \cdot f\right)+\phi \cdot n .
$$


We would now like to estimate the decay of the solution as $|x| \rightarrow \infty$. The first term is easy as it fits into the set up of Lemma C.1, but the second term has to be treated carefully. On the face of it it only decays like $|x|^{-2}$, and we cannot apply Lemma C.1. However the situation is in fact much better. To see this notice that if $u$ is the solution of

$$
\tau^{j} \partial_{j} u=\phi \cdot n
$$

then applying $\tau^{i} \partial_{i}$ to this we get:

$$
-\Delta u=\tau^{i}\left(\nabla_{a}\right)_{i} \phi \cdot n \equiv F \text { since } \mathscr{D}_{\Psi_{0}} n=0 .
$$

Now in this Poisson equation the inhomogeneous term $F=O\left(|x|^{-4}\right)$, and also each component is a derivative since $F=\tau^{i} \partial_{i}(\phi \cdot n)$. Therefore by Fubini we can write the integral as an iterated integral and deduce that the integral of $F$ is zero. This allows us to apply Proposition 6.4.6 in [JT82] to deduce that

$$
|u| \leqq c(1+|x|)^{-2+r}
$$

for any $r \in(0,1)$. Combining this with the estimates in cases A and B of Lemma C.1, we find that $\phi \cdot f$ is square integrable and the result is proved.

The next result is from [AH88]:

Theorem A.11. Given an infinite sequence of $k$-monopoles $\Psi_{0}^{i}$, there exists a partition $k=\sum_{\alpha=1}^{l} k_{\alpha}$ with $k_{\alpha}>0$, sequences of points $x_{i}^{\alpha}$ and gauge transformations $g_{i}^{\alpha} \in C^{\infty}\left(\mathbf{R}^{3} ; S U(2)\right)$ such that

(i) $\rho\left(g_{i}^{\alpha}\right)\left(\Psi_{0}^{i}\left(\cdot-x_{i}^{\alpha}\right)\right.$ converges, subsequently, to a $k_{i}$-monopole in the following sense - on any compact subset of $\mathbf{R}^{3}$ all derivatives converge uniformly.

(ii) As $i \rightarrow \infty$ the distance between $x_{i}^{\alpha}$ and $x_{i}^{\beta}$ tends to $\infty$ while the direction of the line joining $x_{i}^{\alpha}$ to $x_{i}^{\beta}$ converges to a fixed direction.

The proof ([AH88, p. 24]) of this uses the compactness theorems of Uhlenbeck ([Uh182]) in the form given in [Tau82, Part one, Theorem 5.6] and the argument given in [Tau85, p. 527]. The following corollary tells us that there is a one-to-one correspondence between the zero modes of $\Psi_{0}^{i}$ and those of the $k_{\alpha}$-monopoles into which it breaks up for large $i$ :

Corollary A.12. In the situation of the previous theorem let $P^{i}=\rho\left(g_{i}^{\alpha}\right)\left(\Psi_{0}^{i}\left(\cdot-x_{i}^{\alpha}\right)\right.$ and let $Q$ be the $k_{1}$-monopole whose existence is assured. Let $\left\{q_{\mu}\right\}_{\mu=0}^{4 k_{1}-1}$ be an orthonormal basis for the zero modes of $Q$. Let $\rho_{R}$ be the cut-off function defined as follows: $\rho$ is a smooth, positive, radially symmetric, bump function equal to one for $|x| \leqq \frac{1}{2}$ and decreasing monotonically to zero for $|x| \geqq 1$. Now rescale $\rho_{R}(x)=\rho(x / R)$. Then for large $R$ the projection in $H_{P^{i}}$ of the $\rho_{R} q_{\mu}$ onto the space of zero modes of $P_{i}$ has dimension $4 k_{1}$.

Proof. As in [Tau83, Lemma 9.11] we look for a zero mode for $P^{i}$ of the form

$$
r_{\mu}^{i}=\rho_{R} q_{\mu}+\mathscr{D}_{P i}^{*} v_{\mu}^{i},
$$

so that $v_{\mu}^{i}$ satisfies the equation

$$
\mathscr{D}_{P^{i}} \mathscr{D}_{P^{l}}^{*} v_{\mu}^{i}=-\mathscr{D}_{P^{i}}\left(\rho_{R} q_{\mu}\right)=-\rho_{R}\left(\mathscr{D}_{P^{i}}-\mathscr{D}_{Q}\right) q_{\mu}-\tau^{k} \partial_{k} \rho_{R} q_{\mu} .
$$


The idea is to show that $\left|v_{\mu}^{i}\right|_{P^{i}}$ is small for large $i$ and therefore the $r_{\mu}^{i}$ are linearly independent, since for large $R$ the $\rho_{R} q_{\mu}$ are. There exists a solution to this equation which goes to zero as $|x| \rightarrow \infty$ by Theorem A.8. This is true for any $i$ - however there is nothing to guarantee the linear independence of the $r_{\mu}^{i}$ yet. This is where we use the previous theorem. The basic estimate for the previous equation gives us

$$
\begin{aligned}
\left|v_{\mu}^{i}\right|_{P^{i}}^{2} & \leqq\left|\int_{R / 2 \leqq|x| \leqq R}\left(\mathscr{D}_{P^{i}}^{*} v_{\mu}^{i}, \rho R q_{\mu}\right)\right|+\left|\int_{|x| \leqq R / 2}\left(v_{\mu}^{i}, \mathscr{D}_{P^{i}} q_{\mu}\right)\right| \\
& \leqq\left(\int_{R / 2 \leqq|x| \leqq R}\left|q_{\mu}\right|^{2}\right)^{1 / 2}\left|v_{\mu}\right|_{P^{i}}+\left|\int_{|x| \leqq R / 2}\right|\left(\mathscr{D}_{P^{i}}-\mathscr{D}_{Q}\right) q_{\mu}\left\|v_{\mu}\right\| \\
& \leqq\left(\frac{c}{R}+c(R) \max _{|x| \leqq} \frac{R}{2}\left|P^{i}-Q\right|\right)\left|v_{\mu}^{i}\right|_{P^{i}}
\end{aligned}
$$

where the last line uses $|q|=O\left(|x|^{-2}\right)$, and the Poincare and Kato inequalities. Now let $\varepsilon$ be any positive number. Then the first term in brackets can be made less than $\varepsilon / 2$ for large $R$. Fix this value of $R$, then the second term can be made less than $\varepsilon / 2$ by choosing $i$ sufficently large on account of the uniform convergence of $P^{i}$ to $Q$. We therefore deduce that for large $i,\left|v_{\mu}^{i}\right|_{P^{i}}$ gets small and therefore if $q_{\mu}$ is an orthonormal basis then $r_{\mu}^{i}$ are linearly independent for large $i$ since

$$
\left(r_{\mu}^{i}, r_{v}^{i}\right)_{P}^{i}=\delta_{\mu v}+\varepsilon(i, R)_{\mu v},
$$

where $\varepsilon(i, R)_{\mu v} \rightarrow 0$ as $i \rightarrow \infty$ and $R \rightarrow \infty$. This completes the proof.

\section{B. Appendix B: The Error Terms}

If we write the equation for $\psi$ in the form

$$
\psi_{t t}+\mathscr{D}^{*} \mathscr{D} \psi=-\ddot{\Psi}_{0}-\varepsilon \mathscr{D}\left(\partial_{t} \tilde{a}_{0}\right)+\varepsilon^{2} j^{\prime}
$$

We write $j=\left(j_{1}, j_{2}, j_{3}, j_{4}\right)$ with, for $i=1,2,3$,

$$
\begin{aligned}
j_{i}= & -2\left[\tilde{a}_{0}, \dot{\alpha}_{i}\right]+\left[\tilde{a}_{j},\left[\tilde{a}_{j}, \alpha_{i}\right]\right]+\left[\tilde{a}_{j},\left(\nabla_{a}\right)_{j} \tilde{a}_{i}\right]+\left(\nabla_{a}\right)_{j}\left(\left[\tilde{a}_{j}, \tilde{a}_{i}\right]\right) \\
& -\varepsilon\left[\tilde{a}_{0}, \partial_{t} \tilde{a}_{i}\right]-\varepsilon \partial_{t}\left(\left[\tilde{a}_{0}, \tilde{a}_{i}\right]\right)-\varepsilon^{2}\left[\tilde{a}_{0},\left[\tilde{a}_{0}, \alpha_{i}\right]\right] \\
& +\varepsilon^{2}\left[\tilde{a}_{j},\left[\tilde{a}_{j}, \tilde{a}_{i}\right]\right]+\varepsilon^{2}\left[\tilde{a}_{0}, \partial_{i} \tilde{a}_{0}\right]-\varepsilon^{4}\left[\tilde{a}_{0},\left[\tilde{a}_{0}, \tilde{a}_{i}\right]\right] \\
j_{4}= & -2\left[\tilde{a}_{0}, \dot{\varphi}\right]+\left[\tilde{a}_{i},\left[\tilde{a}_{i}, \varphi\right]\right]+\left[\tilde{a}_{i},\left(\nabla_{a}\right)_{i} \tilde{\phi}\right]+\left(\nabla_{a}\right)_{j}\left(\left[\tilde{a}_{j}, \tilde{\phi}\right]\right) \\
& -\varepsilon\left[\tilde{a}_{0}, \tilde{\phi}_{t}\right]+\varepsilon^{2}\left[\tilde{a}_{i},\left[\tilde{a}_{i}, \tilde{\phi}\right]\right]
\end{aligned}
$$

If we write the equation for $\tilde{a}_{0}$ as

$$
-\left(\nabla_{a}\right)_{i}\left(\nabla_{a}\right)_{i} \tilde{a}_{0}+\left[\varphi,\left[\tilde{a}_{0}, \varphi\right]\right]+2\left[\tilde{a}_{i}, \dot{\alpha}_{i}\right]+2[\tilde{\phi}, \dot{\varphi}]=\varepsilon j_{0},
$$

then the error term $j_{0}$ is given by

$$
\begin{aligned}
j_{0}= & -\left[\tilde{a}_{i}, \tilde{a}_{i, t}\right]-\left[\tilde{\phi}_{i}, \tilde{\phi}_{t}\right]+\varepsilon\left[\tilde{a}_{i},\left(\nabla_{a}\right)_{i} \tilde{a}_{0}\right]+\varepsilon\left(\nabla_{a}\right)_{i}\left(\left[\tilde{a}_{i}, \tilde{a}_{0}\right]\right)-\varepsilon\left[\tilde{\phi},\left[\tilde{a}_{0}, \varphi\right]\right] \\
& \left.+\varepsilon\left[\varphi,\left[\tilde{a}_{0}, \tilde{\phi}\right]\right]-\varepsilon^{3}\left[\tilde{\phi},\left[\tilde{a}_{0}, \tilde{\phi}\right]\right]+\varepsilon^{3}\left[\tilde{a}_{i}, \tilde{a}_{0}\right]\right]
\end{aligned}
$$


We need the following estimates for the error terms:

Lemma B.1. Assume that $\left(\psi, \psi_{t}\right) \in H_{3, \Psi_{0}} \oplus H_{2, \Psi_{0}}$ and $\left(\tilde{a}_{0}, \tilde{a}_{0}, t\right) \in H_{4, \Psi_{0}} \oplus H_{3, \Psi_{0}}$ then:

$$
\begin{gathered}
\left|\mathscr{D}\left(\partial_{t} \tilde{a}_{0}\right)\right|_{L^{2}} \leqq\left|\partial_{t} \tilde{a}_{0}\right|_{\Psi_{0}} \\
|j|_{L^{2}}+\left|j_{0}\right|_{L^{2}} \leqq c\left(\left|\tilde{a}_{0}\right|_{2, \Psi_{0}},\left|\tilde{a}_{0, t}\right| \Psi_{0},|\psi|_{\Psi_{0}},|\psi|_{L^{\infty}, \mid},\left|\psi_{t}\right|_{\Psi_{0}},\left|\dot{\Psi}_{0}\right|_{\Psi_{0}},\right. \\
\left|\nabla_{a} j\right|_{L^{2}}+\left|\nabla_{a} j_{0}\right|_{L^{2}} \leqq c\left(\left|\tilde{a}_{0}\right|_{3, \Psi_{0}},\left|\tilde{a}_{0, t}\right|_{2, \Psi_{0}},|\psi|_{2, \Psi_{0}},|\psi|_{L^{\infty}, \mid},\left|\psi_{t}\right|_{2, \Psi_{0}},\left|\dot{\Psi}_{0}\right|_{2, \Psi_{0}}\right) \\
\left|\left(\nabla_{a}\right)_{i}\left(\nabla_{a}\right)_{j} j\right|_{L^{2}}+\left|\left(\nabla_{a}\right)_{i}\left(\nabla_{a}\right)_{j} j_{0}\right|_{L^{2}} \\
\leqq c\left(\left|\tilde{a}_{0}\right|_{4, \Psi_{0}},\left|\tilde{a}_{0, t}\right|_{3, \Psi_{0}}|\psi|_{3, \Psi_{0}},|\psi|_{L^{\infty}, \mid},\left|\psi_{t}\right|_{3, \Psi_{0}},\left|\dot{\Psi}_{0}\right|_{3, \Psi_{0}}\right) \\
\left|j_{0}\right|_{*, \Psi_{0}} \leqq c\left(\left|\psi_{t}\right|_{L^{2}},|\psi|_{\Psi_{0}}+\varepsilon\left|\tilde{a}_{0}\right|_{\Psi_{0}} .\right.
\end{gathered}
$$

Proof. We use Holder's inequality and Lemma A.4. The only subtle point is that in the use of Lemma A.4 we lose a derivative so that we need to do something different to estimate those terms with the highest number of derivatives. What we do is use Sobolev's theorem which ensures that $\psi, \tilde{a}_{0} \in L^{\infty}$ and then estimate

$$
\left|\left[\left(\nabla_{a}\right)_{j} \tilde{a}_{i}, \tilde{a}_{j}\right]\right|_{L^{2}} \leqq\left|\tilde{a}_{j}\right|_{L^{2}}\left|\left(\nabla_{a}\right)_{j} \tilde{a}_{i}\right|_{L^{2}},
$$

and similarly for the other terms.

\section{Appendix C: The Green Function Estimates}

In this section we derive estimates for the Green function for the operator $\tau^{j} \partial_{j}$. This Green function $G(x, y)$ is easily seen to be

$$
G(x, y)=-\frac{\tau^{j}\left(x_{j}-y_{j}\right)}{4 \pi|x-y|^{3}} .
$$

This means that $\tau^{i} \partial_{i} G(x, y)=-\delta_{y}$.

Lemma C.1. Let $u, f$ be smooth quaternion valued functions which decay uniformly to zero as $|x| \rightarrow \infty$ and satisfy

$$
\tau^{j} \partial_{j} u=f
$$

then

Case A. If $f=g h$ where $|g| \in L^{6}$ and

$$
|h(x)| \leqq \frac{M}{(1+|x|)^{2}},
$$

then for any positive $\varepsilon$ there is a number $c\left(\varepsilon,|g|_{L^{6}}, M\right)$ such that

$$
|u(x)| \leqq \frac{c}{(1+|x|)^{3 / 2-\varepsilon}} .
$$


Case $B$.

$$
|f(x)| \leqq \frac{M}{(1+|x|)^{7 / 2-\varepsilon}},
$$

then there exists $c=c(M)$ such that

$$
|u(x)| \leqq \frac{c(M)}{(1+|x|)^{2}} .
$$

Case $C$.

$$
\int f(y) d y=0 \text { and }|f(x)| \leqq \frac{M}{(1+|x|)^{4}},
$$

then for any positive $\varepsilon$ there is a number $c=c(M, \varepsilon)$ such that

$$
|u(x)| \leqq \frac{c(M, \varepsilon)}{(1+|x|)^{3-\varepsilon}}
$$

Remark. No attempt has been made to be either general or sharp in this lemma.

Proof. In all three cases we will split up the integral into an inner region $I=$ $\left\{y:|x-y|<\frac{|x|}{2}\right\}$ and an outer region $O=\left\{y:|x-y|>\frac{|x|}{2}\right\}$. Notice that on account of the inequality

$$
\|a|-| a-b\| \leqq|b|
$$

$|y|>\frac{|x|}{2}$ in the inner region. For Case A we estimate the integrals as follows:

$$
\begin{aligned}
|u(x)| \leqq & \int_{I} \frac{|g||h| d y}{|x-y|^{2}}+\int_{O} \frac{|g||h| d y}{|x-y|^{2}} \\
\leqq & \frac{c}{(1+|x|)^{2}}|g|_{L^{6}}\left(\int_{I}|x-y|^{-12 / 5} d y\right)^{5 / 6} \\
& +|g|_{L^{6}}|h|_{L^{p}}\left(\int_{O}|x-y|^{-2 q} d y\right)^{\frac{1}{q}}
\end{aligned}
$$

where we choose $p=3 / 2+\delta$ and

$$
\frac{1}{6}+\frac{1}{p}+\frac{1}{q}=1
$$

For sufficiently small $\delta$ this will give the result. For Case B we proceed similarly:

$$
\begin{aligned}
|u(x)| & \leqq \int_{I} \frac{|f| d y}{|x-y|^{2}}+\int_{O} \frac{|f| d y}{|x-y|^{2}} \\
& \leqq \frac{M}{(1+|x|)^{7 / 2-\varepsilon}} \int_{I}|x-y|^{-2} d y+\frac{4}{|x|^{2}} \int_{O}|f(y)| d y \\
& \leqq \frac{c}{|x|^{2}}
\end{aligned}
$$


Finally for Case $\mathrm{C}$ we take advantage of the fact that $\int f=0$ to write the solution as

$$
u(x)=(4 \pi)_{\tau^{j}}^{-1}\left(\int_{1} f(y)\left(\frac{x_{j}-y_{j}}{|x-y|^{3}}-\frac{x_{j}}{|x|^{3}}\right) d y+\int_{O} f(y)\left(\frac{x_{j}-y_{j}}{|x-y|^{3}}-\frac{x_{j}}{|x|^{3}}\right) d y\right) .
$$

The inner integrals can both be easily estimated as $\leqq \frac{c}{(1+|x|)^{3}}$ since $|y| \geqq|x| / 2$ in the inner region. For the outer integral we use the following identity:

$$
\begin{aligned}
\left(x_{j}-y_{j}\right)|x|^{3}-x_{j}|x-y|^{3} \equiv & \left(x_{j}-y_{j}\right)|x|^{2}(|x|-|x-y|)+2 x \cdot y|x-y|\left(x_{j}-y_{j}\right) \\
& -x_{j}|y|^{2}|x-y|+y_{j}|x-y|\left(2 x \cdot y-|x|^{2}\right) .
\end{aligned}
$$

This allows us to estimate the outer integral as

$$
\begin{aligned}
& \leqq \frac{c}{|x|} \int_{O} \frac{|y \| f(y)| d y}{|x-y|^{2}}+\frac{c}{|x|^{2}} \int_{O} \frac{|y \| f(y)| d y}{|x-y|}+\frac{c}{|x|^{2}} \int_{O} \frac{|y|^{2}|f(y)| d y}{|x-y|^{2}} \\
& \leqq \frac{c}{|x|^{3-3 / p}}\left(|y f(y)|_{L^{q}}+\frac{c}{|x|^{4-3 / p^{\prime}}}\right),
\end{aligned}
$$

where

$$
q>1, \frac{1}{p}+\frac{1}{q}=1 \text { and } q^{\prime}>\frac{3}{2}, \frac{1}{p^{\prime}}+\frac{1}{q^{\prime}}=1 .
$$

Now we choose $p$ large and $3-p^{\prime}$ small to obtain the desired estimate.

\section{Appendix D: Asymptotic Behaviour of Monopoles}

In this section we give a proof of an assertion in [Hit83] which is attributed to unpublished work of Taubes. The proof is an application of the ideas in [JT82].

Theorem D.1. Finite action solutions with monopole number $k$ of the Bogomolny equations with boundary conditions

$$
|\phi| \rightarrow 1 \text { as }|x| \rightarrow \infty
$$

automatically satisfy the following boundary conditions (for any $\varepsilon>0$ ) :

$$
\begin{aligned}
|D \phi| & =\frac{k}{|x|^{2}}+O\left(|x|^{-3+\varepsilon}\right), \\
|\phi| & =1-\frac{k}{|x|}+O\left(|x|^{-2}\right), \\
\frac{\partial|\phi|}{\partial \Omega} & =O\left(|x|^{-2}\right) .
\end{aligned}
$$

Proof. To start with it is proved in [JT82] that $|D \phi|=O\left(|x|^{-2}\right)$. To improve this to the first statement we use the identity 


$$
\Delta w=-g \text { where } w=\frac{1}{2}\left(1-|\phi|^{2}\right) g=|D \phi|^{2}
$$

from which we obtain the following formula:

$$
\nabla w=\frac{k x}{|x|^{3}}+\int\left(\frac{x-y}{|x-y|^{3}}-\frac{x}{|x|^{3}}\right) g(y) d y .
$$

Then since $|g|=O\left(|y|^{-4}\right)$ we can apply case C of Theorem C.1 to obtain

$$
\nabla w=\frac{k x}{|x|^{3}}+O\left(|x|^{-3+\varepsilon}\right)
$$

but since $\nabla w=-(\phi, D \phi)$ and the transverse component of $D \phi$ is exponentially decaying this gives the result.

To obtain the second statement we notice that since $\int|D \phi|^{2}=4 \pi k$ we can write

$$
w=\frac{k}{2|x|}+\int\left(\frac{1}{|x|}-\frac{1}{|x-y|}\right) g(y) d y .
$$

Now we know that

$$
g=|D \phi|^{2}=\frac{k^{2}}{|x|^{-4}}+O\left(|x|^{-5+\varepsilon}\right)
$$

We treat these two terms separately - for the radial term $\frac{k^{2}}{|x|^{-4}}$ we can solve the Poisson equation explicitly, to find it contributes $\frac{1}{2} k^{2}|x|^{-2}$ to $w$. For the second term we can apply Theorem 6.4 .6 of [JT82] to show that this also contributes a term of $O\left(|x|^{-2}\right)$ to $w$. This completes the proof of the second statement.

Finally for the third statement we have to consider the effects of angular derivatives $\Omega=x_{1} \partial_{2}-x_{2} \partial_{1}$. We use Eq. D again, and the formula

$$
g=\frac{k^{2}}{|x|^{4}}+O\left(|x|^{-5+\varepsilon}\right)
$$

and since we may evaluate directly the effect of the radial term we may assume that $g=O\left(|x|^{-5+\varepsilon}\right)$. But then an examination of the proof of Case C of Theorem C. 1 shows that the second term in the formula for $\nabla w$ contributes $O\left(|x|^{-3}\right)$. Finally taking angular derivatives we find the effect of the first term vanishes:

$$
\frac{x_{1} x_{2}}{|x|^{3}}-\frac{x_{2} x_{1}}{|x|^{3}}=0
$$

and we end up with the result we wanted.

\section{E. Appendix E: The Local Existence Theorem}

In this section we explain how to prove the local existence theorem. The approach is via a very standard iteration scheme, and since the method is very similar to an analagous proof in [Stu] we will be brief. We want to construct solutions of the system of equations: 


$$
\begin{gathered}
\psi_{t t}+L_{\Psi_{0}} \psi=\dot{\Psi}_{0}-\varepsilon \mathscr{D} \Psi_{0}\left(\partial_{t} \tilde{a}_{0}\right)+\varepsilon^{2} j^{\prime} \\
-\Delta_{\alpha} \tilde{a}_{0}+\left[\varphi,\left[\tilde{a}_{0}, \varphi\right]\right]=2\left[\dot{\alpha}_{k}, \tilde{a}_{k}\right]+\varepsilon j_{0} \\
\frac{d Z}{d t}-\varepsilon F\left(q^{0}, Z, \psi, \mathscr{D}\left(\partial_{t} \tilde{a}_{0}\right)\right) .
\end{gathered}
$$

To solve these we set up an iteration scheme as follows: for initial data for $\psi^{(i)}$ iterate we take a smooth compactly supported approximation of $\psi(0, x), \psi_{t}(0, x)$ such that

$$
\left\|\psi^{(i)}(0)-\psi(0)\right\| \leqq \delta 2^{-i}
$$

where $\delta$ can be taken arbitrarily small, while for $Z^{(i)}$ we take

$$
Z^{(i)}(0)=Z(0)
$$

For the initial iterate we take

$$
\psi^{(0)}(t, x)=\psi^{(0)}(0, x), \quad \tilde{a}_{0}^{(0)}(t, x)=0, \quad Z^{(0)}(t)=Z(0) .
$$

We then produce successive approximations by solving the equations

$$
\begin{aligned}
& -\Delta_{a(0)} \tilde{a}_{0}^{(i+1)}+\left[\phi(0),\left[\tilde{a}_{0}^{(i+1)}, \phi(0)\right]\right]=2 \sum_{k}\left[\alpha_{k}^{(i)}, \tilde{a}_{k}^{(i)}\right] \\
& +\left(\Delta_{\alpha^{(i)}}-\Delta_{a(0)}\right) \tilde{a}_{0}^{(i)}+\left[\varphi^{(i)},\left[\tilde{a}_{0}^{(i)}, \varphi^{(i)}\right]\right]-\left[\phi(0),\left[\tilde{a}_{0}^{(i)}, \phi(0)\right]\right] \\
& +\varepsilon j_{0}\left(\psi^{(i)}, \tilde{a}_{0}^{(i)}, \Psi_{0}^{(i)},\right. \\
& \psi_{t t}^{(i+1)}-\Delta_{a(0)} \psi^{(i+1)}+\left[\phi(0),\left[\psi^{(i+1)}, \phi(0)\right]\right]=-\ddot{\Psi}_{0}^{(i)}+\left(\Delta_{\alpha^{(i)}}-\Delta_{a(0)}\right) \psi^{(i)} \\
& +\left[\varphi^{(i)},\left[\psi^{(i)}, \varphi^{(i)}\right]\right]-\left[\phi(0),\left[\psi^{(i)}, \phi(0)\right]\right] \\
& -\varepsilon \mathscr{D}_{\Psi_{0}^{(i)}}\left(\partial_{t} \tilde{a}_{0}^{(i+1)}\right)+\varepsilon^{2} j^{\prime}\left(\tilde{a}_{0}^{(i+1)}, \psi^{(i)}, \Psi_{0}^{(i)},\right. \\
& \frac{d Z^{(i+1)}}{d t}=\varepsilon F^{(i)}\left(q^{0}, Z^{(i)}, \psi^{(i)}, \mathscr{D}\left(\tilde{a}_{0}^{(i+1)}\right)\right),
\end{aligned}
$$

where $\Psi_{0}^{(i)}=\left(\alpha^{(i)}, \varphi^{(i)}\right)=\Psi_{0}\left(q^{(0)}+\varepsilon Z^{(i)}\right)$. The first equation is solved by means of Theorem A.8, while for the second we need the following:

Lemma E.1. Let $\Psi_{0}=(a, \phi)$ be a fixed monopole configuration, and consider the equation

$$
\psi_{t t}-\Delta_{a} \psi+[\phi,[\psi, \phi]]=f
$$

Assume that

$$
\int_{\mathbf{R}^{3}}|f|^{2}+\sum_{i}\left|\left(\nabla_{a}\right)_{i} f\right|^{2}+\sum_{i, j}\left|\left(\nabla_{a}\right)_{i}\left(\nabla_{a}\right)_{j} f\right|^{2}<\infty
$$

and that $t \rightarrow f(t)$ is continuous with respect to the topolgy defined by these norms. Assume that the initial data is smooth and compactly supported. Then there exists a solution which satisfies 


$$
\begin{aligned}
& \max _{0 \leqq t \leqq T}\left(|\psi(t)|_{3, \Psi_{0}}+\left|\psi_{t}(t)\right|_{2, \Psi_{0}}\right) \\
& \leqq \\
& \quad|\psi(0)|_{3, \Psi_{0}}+\left|\psi_{t}(0)\right|_{2, \Psi_{0}}+c \int_{0}^{T}\left(|f|^{2}+\sum_{i}\left(|f|^{2}+\sum_{i}\left|\left(\nabla_{a}\right)_{i} f\right|^{2}\right.\right. \\
& \left.\quad+\sum_{i, j}\left|\left(\nabla_{a}\right)_{i}\left(\nabla_{a}\right)_{j} f\right|^{2}\right)^{1 / 2} d x d t+c \int_{0}^{T}\left(|\psi(0)|_{3, \Psi_{0}}+\left|\psi_{t}(0)\right|_{2, \Psi_{0}}\right) d t
\end{aligned}
$$

Proof. See Sect. 3(b) of Chapter Five of reference [Joh81] for example. In applying $\left(\nabla_{a}\right)_{i}$ to the equation to obtain the higher order energies commutator terms appear which is what makes the final term on the right-hand side necessary.

The iterates are now well defined. The crucial step in proving the local existence theorem is obtaining uniform bounds on the iterates. Once this is done it is straightforward to show that the iterates are a Cauchy sequence.

Step One. Uniform Boundedness. The uniform bounds come from the following result:

Claim. Given a positive number $\Gamma$, there exist numbers $A(\Gamma), B(\Gamma)$ such that the following is true: assume that for $j \leqq i$ we have bounds

$$
\left\|\psi^{(j)}(T)\right\| \leqq \Gamma,\left\|\tilde{a}_{0}^{(j)}(T)\right\| \leqq 2 A(\Gamma),\left\|Z^{(j)}(T)\right\| \leqq \Gamma, \max _{0 \leqq t \leqq T}\left|\psi_{t t}^{(j)}\right|_{1, \Psi_{0}} \leqq 2 B(\Gamma),
$$

then for the $(i+1)^{\text {th }}$ iterate we have

$$
\begin{gathered}
\left\|\psi^{(i+1)}(T)\right\| \leqq \frac{\Gamma}{2}+\delta+T c(\Gamma), \\
\left\|\tilde{a}_{0}^{(i+1)}(T)\right\| \leqq A(\Gamma)\left(1+\varepsilon c(\Gamma)\left|\psi_{t t}^{(i)}\right|_{1, \Psi_{0(0)}}\right), \\
\max _{0 \leqq t \leqq T}\left|\psi_{t t}^{(i+1)}\right|_{1, \Psi_{0(0)}} \leqq B(\Gamma)\left(1+\varepsilon\left\|\tilde{a}_{0}^{(i+1)}(T)\right\|\right), \\
\|Z(T)\| \leqq \frac{\Gamma}{2}+\varepsilon T c(\Gamma) .
\end{gathered}
$$

Proof of Claim. Let us consider first the $\tilde{a}_{0}$ equation. From our assumptions on the previous iterates we can estimate the $O(\varepsilon)$ term by:

$$
\varepsilon\left|j_{0}(i)\right|_{\Psi_{0}^{*}}+\varepsilon\left|j_{0}(i)\right|_{L^{2}}+\varepsilon\left|j_{0}(i)\right|_{2, \Psi_{0}} \leqq \varepsilon c(\Gamma) .
$$

By our assumptions on $Z^{j}, j \leqq i$ we have an estimate of the form

$$
\left|\Psi_{0}^{(i)}-\Psi_{0}(0)\right|_{L^{2}}+\left|\Psi_{0}^{(i)}-\Psi_{0}(0)\right|_{r, \Psi_{0}(0)} \leqq \varepsilon t c(\Gamma),
$$

from which we can estimate the term

$$
\left(\Delta_{\alpha}^{(i)}-\Delta_{a(0)}\right) \tilde{a}_{0}^{(i)}+\left[\varphi^{(i)},\left[\tilde{a}_{0}^{(i)}, \varphi^{(i)}\right]\right]-\left[\phi(0),\left[\tilde{a}_{0}^{(i)}, \phi(0)\right]\right] .
$$

Finally we have a bound for the norm of $2 \sum_{k}\left[\alpha_{k}^{(i)}, \tilde{a}_{k}^{(i)}\right]$ which gives the required estimate for $\tilde{a}_{0}^{(i+1)}$. In an identical fashion we get the other estimates. The estimate for $\psi_{t t}$ comes from the equation. Now by choosing $\varepsilon, T$ sufficently small we find that 
the $(i+1)^{\text {th }}$ iterates obey the bounds in E.1. These bounds imply further bounds by differentiation with respect to time. This gives uniform bounds for $\left|\psi_{t t t}\right|_{L^{2}}$ and $\left|\tilde{a}_{0, t}^{(i)}\right|_{2, \Psi_{0}}$ and $\ddot{Z}^{(i)}$.

Step Two. Convergence of the iterates. Having obtained these uniform bounds the convergence of the iterates in the norm

$$
\left\|\psi^{(i)}\right\|_{1-}+\left\|\tilde{a}_{0}(i)\right\|_{2}+\left\|Z^{(i)}\right\|_{3}+\max _{0 \leqq t \leqq T}\left|\psi_{t t}^{(i)}\right|_{1, \Psi_{0}(0)}
$$

follows in the usual way since the inhomogeneuous terms are Lipshitz with respect to the norms $\|\cdot\|_{i}$ (see e.g. [Stu].) By using the equations and their time derivatives we can deduce from this the convergence of the iterates measured by

$$
\max _{0 \leqq t \leqq T}\left\|\tilde{a}_{0, t t}^{(i)}\right\|_{1, \Psi_{0}(0)}+\left\|\dot{Z}^{(i)}\right\|_{3}+\left\|\ddot{Z}^{(i)}\right\|_{3} \text {. }
$$

Step Three. Properties of Solution. The uniform bounds on the higher time derivatives then ensure the stated regularity properties with respect to time. By writing down the corresponding versions of the energy identities of Sect. 6 for the iterates, and then taking the limit, we deduce that the solution satisfies those identities. Direct calculation shows that the gauge orthogonality condition is preserved by the equations, as are the condition $\left(\psi, n_{\mu}\right)_{L^{2}}=0$. (Indeed the equation for $\dot{Z}$ was chosen exactly such that this is so!). Notice that while we do not prove estimates for $|\psi|_{L^{2}}$, we know that as long as the initial data is square integrable then so is $\psi$ since

$$
|\psi(t)|_{L^{2}} \leqq|\psi(0)|_{L^{2}}+\int_{0}^{T}\left|\psi_{t}(t)\right|_{L^{2}} d t .
$$

Thus the condition $\left(\psi, n_{\mu}\right)_{L^{2}}=0$ makes sense.

\section{References}

[AH88] Atiyah, M., Hitchin, N.: Geometry and Dynamics of Magnetic Monopoles. Princeton, NJ: Princeton University Press, 1988

[Ben72] Benjamin, T.B.: Stability of solitons. Proceedings of the Royal Society A328, 153-179 (1972)

[Ble81] Bleecker, D.: Gauge theory and variational principles. Reading, Mass: Addison Wesley, 1981

[CL84] Cheng, T.P., Li, L.F.: Gauge Theory of Elementary Particle Physics. Oxford: Oxford University Press, 1984

[Don84] Donaldson, S.: Nahm's equations and the classification of monopoles. Commun. Math. Phys. 96, 387-407 (1984)

[ES89] Ercolani, N., Sinha, A.: Monopoles and Baker functions. Commun. Math. Phys. 125, 385-416 (1989)

[Gro84] Groisser, D.: Integrality of the monopole number. Commun. Math. Phys. 93, 367-378 (1984)

[Hit82] Hitchin, N.: Monopoles and geodesics. Commun. Math. Phys. 83, 579-602 (1982)

[Hit83] Hitchin, N.: On the construction of monopoles. Commun. Math. Phys. 89, 145-190 (1983)

[Joh81] John, F.: Partial Differential Equations. New York: Springer, 1981

[JT82] Jaffe, A., Taubes, C.: Vortices and Monopoles. Boston, Mass: Birkhauser, 1982

[Mai81] Maison, D.: Uniqueness of the Prasad Sommerfeld monopole solution. Nucl. Phys. B 182, 114-124 (1981) 
[Man82] Manton, N.: A remark on scattering of BPS monopoles. Phys. Lett. 110B, 54-56 (1982)

[MB88] Montgomery, R., Bates, L.: Closed geodesics on the space of stable two monopoles. Commun. Math. Phys. 118, 635-640 (1988)

[Stu] Stuart, D.: Dynamics of Abelian Higgs vortices in the near Bogomolny regime. Commun. Math. Phys. 159, 51-91 (1994)

[Tau82] Taubes, C.: Existence of a non-minimal solution to SU(2) Yang-Mills equations on $\mathbf{R}^{3}$, Parts one and two. Commun. Math. Phys. 86, 257-320 (1982)

[Tau83] Taubes, C.: Stability in Yang-Mills theories. Commun. Math. Phys. 91, 235-263 (1983)

[Tau85] Taubes, C.: Min-max theory for Yang-Mills-Higgs. Commun. Math. Phys. 97, 473-540 (1985)

[Uhl82] Uhlenbeck, K.: Connections with $1^{p}$ bounds on Curvature. Commun. Math. Phys. 83, 31-42 (1982)

[Wei79] Weinberg, E.: Parameter counting for multi-monopole solutions. Phys. Rev. D 20, 936944 (1979)

Communicated by A. Jaffe 